\title{
QUEEN'S
UNIVERSITY
BELFAST
}

\section{A strongly degenerate diffusion-haptotaxis model of tumour invasion under the go-or-grow dichotomy hypothesis}

Zhigun, A., Surulescu, C., \& Hunt, A. (2018). A strongly degenerate diffusion-haptotaxis model of tumour invasion under the go-or-grow dichotomy hypothesis. Mathematical Methods in the Applied Sciences, 41(6), 2403-2428. https://doi.org/10.1002/mma.4749

Published in:

Mathematical Methods in the Applied Sciences

Document Version:

Peer reviewed version

Queen's University Belfast - Research Portal:

Link to publication record in Queen's University Belfast Research Portal

Publisher rights

(c) 2018 John Wiley \& Sons, Ltd

This work is made available online in accordance with the publisher's policies. Please refer to any applicable terms of use of the publisher.

\section{General rights}

Copyright for the publications made accessible via the Queen's University Belfast Research Portal is retained by the author(s) and / or other copyright owners and it is a condition of accessing these publications that users recognise and abide by the legal requirements associated with these rights.

Take down policy

The Research Portal is Queen's institutional repository that provides access to Queen's research output. Every effort has been made to ensure that content in the Research Portal does not infringe any person's rights, or applicable UK laws. If you discover content in the Research Portal that you believe breaches copyright or violates any law, please contact openaccess@qub.ac.uk. 


\title{
A strongly degenerate diffusion-haptotaxis model of tumor invasion under the go-or-grow dichotomy hypothesis
}

\author{
Anna Zhigun ${ }^{\mathrm{a} *}$, Christina Surulescu ${ }^{\mathrm{a}}$, and Alexander Hunt ${ }^{\mathrm{a}}$
}

\begin{abstract}
We propose and study a strongly coupled PDE-ODE-ODE system modeling cancer cell invasion through a tissue network under the go-or-grow hypothesis asserting that cancer cells can either move or proliferate. Hence our setting features two interacting cell populations with their mutual transitions and involves tissue-dependent degenerate diffusion and haptotaxis for the moving subpopulation. The proliferating cells and the tissue evolution are characterized by way of ODEs for the respective densities. We prove the global existence of weak solutions and illustrate the model behaviour by numerical simulations in a two-dimensional setting. The numerical results recover qualitatively the infiltrative patterns observed histologically, and moreover allow to establish a qualitative relationship between the structure of the tissue and the expansion of the tumor, thereby paying heed to its heterogeneity. Copyright $\odot 20 X X$ John Wiley \& Sons, Ltd.
\end{abstract}

Keywords: cancer cell invasion; degenerate diffusion; global existence; go-or-grow dichotomy; haptotaxis; parabolic system; weak solution

\section{Introduction}

One of the essential characteristics of a tumor is its heterogeneity. The cells forming the neoplastic tissue often have different phenotypes, morphologies, and functions, and can switch between these in response to intra- and/or extracellular influences like e.g., genetic change, acidity of the peritumoral region, availability of nutrients and/or space, applied therapeutic agents etc., see e.g. $[1,2,3]$. Tumor heterogeneity is tightly connected to compromised treatment response $[4,5]$ and is already manifested at the migrating stage of tumor development. Indeed, one of the main features of tumor development and invasion is the ability of cancer cells to migrate and spread into the normal tissue, whereby they experience different migratory phenotypes (e.g., amoeboid vs. mesenchymal). Furthermore, experimental evidence revealed that several types of tumor cells (including glioma, breast cancer cells, and melanoma) defer their proliferation while migrating and vice versa $[6,7,8,9]$, corresponding to the so-called go-or-grow dichotomy. The differentiated response of tumor cells to treatment is a main cause of radio- and chemotherapeutical failure; indeed, it is argely accepted that cells with a highly proliferating phenotype are more sensitive to therapy, whereas the migratory phenotype is attended by reduced treatment sensitivity, see e.g., $[10,11,12]$ and the references therein.

Motivated by the above mentioned facts we propose in this paper a model for tumor cell invasion in which we account for the goor-grow hypothesis and distinguish between migrating and proliferating (hence non-moving) cells. Several continuum mathematical models relying on the go-or-grow behavior of tumor cells and explicitly accounting for the two subpopulations of migrating and proliferating cells, respectively, have been considered e.g., in [13, 14] and featured reaction-(cross-)diffusion(-chemotaxis) equations. Using a two-component continuous-time random walk along with a probabilistic approach based thereupon and involving switching with exponentially distributed waiting times between the proliferation and migration phenotypes, Fedotov \& lomin deduced in [15] an ODE-PDE system for the macroscopic dynamics of the two types of cancer cell densities, supporting the idea of tumor cells subdiffusivity instead of the more common Fickian diffusivity. In [16] Chauviere et al. used a mesoscopic description of the two cell subpopulations to deduce by an appropriate scaling a system of two coupled reaction-diffusion equations for their macrolevel behavior. Still in that context, starting from mesoscopic equations for the two cell subpopulations and coupling them with subcellular level dynamics in $[17,18]$ the authors obtained by parabolic scalings macroscopic equations characterizing the evolution

a Technische Universität Kaiserslautern, Felix-Klein-Zentrum für Mathematik, Paul-Ehrlich-Str. 31, 67663 Kaiserslautern, Germany

* Correspondence to: Technische Universität Kaiserslautern, Fachbereich Mathematik, Postfach 3049,67663 Kaiserslautern, Germany. E-mail: zhigun@mathematik.uni-kl.de 
of the overall tumor burden for a glioma invasion model. The resulting equations carried in their coefficients the information from the lower modeling scales (both subcellular and mesoscopic) and allowed DTI-based predictions about the tumor extent and simulation-based therapy outcomes. The haptotaxis term obtained in those macroscopic equations was a direct consequence of accounting for the subcellular receptor binding dynamics in the mesoscale evolution of the cancer cell densities. By using the equlibrium of fluxes and some ideas from [19, 20], in [21] was introduced a multiscale model for macroscopic tumor invasion and development complying to the go-or-grow dichotomy and including subcellular dynamics of receptor binding to fibers of the underlying extracellular matrix (ECM). Our model in this paper extends in a certain way the previous setting in [21] by allowing the diffusion coefficient to degenerate and by paying increased attention to the haptotactic sensitivity function; however, neither therapy effects nor multiscality issues are addressed here. The system in [22] characterizes the dynamics of two cell populations (quiescent and proliferating) under the influence of haptotaxis, matrix degrading enzymes, and of a diffusing chemical -but without chemotaxis terms- and featuring besides space another structure, namely cell age. Both cell populations diffuse and the diffusion coefficients do not degenerate. Moreover, the interpopulation transition rates are assumed to be smooth, bounded, and nonnegative. Under these and other requirements the global existence of a unique nonnegative solution has been proved. To our knowledge the model introduced and analyzed here is the first to include haptotaxis, strongly -doubly- degenerate diffusion, and interactions between two cell (sub)populations, one of which is neither diffusing nor performing any other kind of motion. Moreover, we do not require the boundedness of interpopulation transition rates and decay coefficient functions. Indeed, some of them depend in a linear way upon a solution component, and the global boundedness of the latter is still open in the context of our model.

While there is a vast literature concerning the mathematical modelling with reaction-diffusion-taxis equations and their analysis, problems with degenerate diffusion and taxis are still comparatively underrepresented in the modelling for life sciences, which is probably to be attributed to their higher complexity. However, during the last decade more such references became available; they describe the dynamics of a cell population in response to a chemoattractant [23, 24, 25], moving up the gradient of an insoluble signal (haptotaxis) $[26,27]$, or performing both chemo- and haptotaxis $[28,29,30]$. Thereby, the type of degeneracy is a particularly relevant feature for the difficulty of the problem, especially for systems coupling ODEs with PDEs, as is the case when considering haptotaxis. In $[28,29,30]$ the diffusion coefficients depend nonlinearly on the solution and the tactic sensitivities are constants. For these problems the global well posedness was obtained, along with boundedness properties of the solutions. The 1D model in [27] was motivated by the deduction of macroscopic equations from a mesoscopic setting for brain tumor invasion also accounting for subcellular dynamics; it features a reaction-diffusion-transport-haptotaxis equation for the tumor cell density coupled with an ODE for the density of tissue fibers. The strong degeneracy of the (myopic) diffusion and haptotaxis coefficients is attained by way of a function only depending on the position and not on the solution itself. The model proposed in [26] involves a diffusion coefficient which can degenerate due to each of the solution components (density of cells and of ECM fibers, respectively): moreover, the haptotactic sensitivity is a nonlinear function of the ECM density. The model we propose and study here extends that in [26] by allowing for tumor heterogeneity in the sense of the go-or-grow dichotomy; this extension is motivated by the biomedical facts mentioned at the beginning of this section. Whereas the global existence of weak solutions was shown for both models in [27] and [26], respectively, the boundedness and uniqueness issues remain open. The same applies to the mathematical setting considered in this work and presented in detail in the following Section 2. The mathematical analysis performed here follows the approach we introduced in [26]. However, the allegedly simple modifications of the model therein lead to mathematical challenges and some new ideas are necessary because in the current system the motility and growth/decay mechanisms are distributed between two separate equations for two distinct cell densities. This leads to an even less regular system than that in [26] which in turn requires even finer tuned estimates as well as a rougher solution concept in order to obtain the global existence.

The rest of the paper is organised as follows: Section 3 introduces some basic notations, Section 4 settles the problem and states the main result consisting in the global existence of a weak solution to the system in Section 2, to be followed by several steps towards its proof. Thus, Section 5 introduces a sequence of non-degenerate approximations of the actual problem and Section 6 is concerned with deducing some apriori estimates to be used in Section 7 for the convergences necessary to prove the result announced in Section 4. Finally, in Section 8 we perform some numerical simulations in order to illustrate the model behavior and we also comment on the obtained results.

\section{The model}

Based on the models in $[21,26]$ we introduce here a PDE-ODE-ODE system characterizing the macroscopic dynamics of a tumor in interaction with the surrounding tissue in accordance with the go-or-grow dichotomy. The latter means that the tumor is assumed to be made up of two types of cells, which are either moving or mitotic and non-motile, whereby mutual transitions between the 
two phenotypes take place. Our model thus reads:

$$
\begin{array}{ll}
\partial_{t} m=-\alpha m+\beta v p+\nabla \cdot\left(\frac{\kappa_{m} v c}{1+v c} \nabla m-\frac{\kappa_{v} m}{(1+v)^{2}} \nabla v\right) & \text { in } \mathbb{R}^{+} \times \Omega, \\
\partial_{t} p=\alpha m-\beta v p+\mu_{p} p(1-c-\eta v) & \text { in } \mathbb{R}^{+} \times \Omega, \\
\partial_{t} v=\mu_{v} v(1-v)-\lambda v m & \text { in } \mathbb{R}^{+} \times \Omega, \\
\frac{\kappa_{m} v c}{1+v c} \partial_{\nu} m-\frac{\kappa_{v} m}{(1+v)^{2}} \partial_{\nu} v=0 & \text { in } \mathbb{R}^{+} \times \partial \Omega, \\
m(0)=m_{0}, p(0)=p_{0}, v(0)=v_{0} & \text { in } \Omega,
\end{array}
$$

where $m$ and $p$ denote the densities of moving and proliferating cells, respectively, $v$ is the density of ECM fibers, all depending on time and position on a smooth bounded domain $\Omega \subset \mathbb{R}^{N}$. The positive constants $\alpha, \beta$ denote the transition rates between the two subpopulations, $\eta>0$ is a constant scaling the concurrence with normal tissue in the proliferation process, $\kappa_{m}, \kappa_{v}$ are positive constants scaling the diffusion and the haptotactic sensitivity, $\lambda>0$ is the decay rate of ECM due to interactions with (mesenchymally) motile cells, and $\mu_{p}, \mu_{v}$ are growth rates for the tumor cells and the tissue, respectively. The total tumor burden is assessed by

$$
c(t, x):=m(t, x)+p(t, x)
$$

Thus, system (1) includes a degenerate parabolic PDE for the moving and an ODE for the proliferating tumor cells, together with an ODE for the tissue density, supplemented by the initial and the 'no-flux' boundary conditions. The latter complies with the fact that cancer cells do not leave the tissue hosting the original tumor. As in [26], the diffusion coefficient in the equation for moving cells is nonlinear and degenerate with respect to both the total tumor cell and tissue densities. On one hand, this type of diffusion captures the infiltrative, finite speed propagation of the cells. On the other hand, it accounts for the necessity for the cells to maintain a strong connection to the tissue fibers in order to be able to move. The haptotaxis coefficient is nonlinear as well; its form is motivated by the microlocal cell-tissue interactions (as explained in [26]) and whence keeps a flavor of multiscality, though in a rather indirect fashion, as our system (1) is purely macroscopic. For explicit multiscale effects and therapy issues we refer to the related model in [21], which, however, only handles nondegenerate diffusion of the moving cell population. Altogether, compared to the existing quasilinear haptotaxis equations, equation ( $1 \mathrm{a}$ ) features less balance between the two flux components (diffusive and haptotactic), with emphasis on the latter due to the degenerate diffusion. This is in agreement with the invasion pattern usually observed histologically, where the directed movement along the tissue fibers dominates over the random dispersion.

\section{Basic notation and functional spaces}

We denote the Lebesgue measure of a set $A$ by $|A|$ and by $\operatorname{int} A$ its interior.

Partial derivatives, in both classical and distributional sense, with respect to variables $t$ and $x_{i}$, will be denoted respectively by $\partial_{t}$ and $\partial_{x_{i}}$. Further, $\nabla, \nabla \cdot$ and $\Delta$ stand for the spatial gradient, divergence and Laplace operators, respectively. $\partial_{\nu}$ is the derivative with respect to the outward unit normal of $\partial \Omega$. We assume the reader to be familiar with the standard Lebesgue and Sobolev spaces and their usual properties, as well as with the more general $L^{p}$ spaces of functions with values in general Banach spaces and with anisotropic Sobolev spaces. In particular, we need the Banach space

$$
\begin{aligned}
& W^{-1,1}(\Omega):=\left\{u \in D^{\prime}(\Omega) \mid u=u_{0}+\sum_{k=1}^{N} \partial_{x_{i}} u_{i} \text { for some } u_{i} \in L^{1}(\Omega), i=0, \ldots, N\right\}, \\
& \|u\|_{W^{-1,1}(\Omega)}:=\inf \left\{\sum_{k=0}^{N}\left\|u_{i}\right\|_{1} \mid u=u_{0}+\sum_{k=1}^{N} \partial_{x_{i}} u_{i}, u_{i} \in L^{1}(\Omega), i=0, \ldots, N\right\} .
\end{aligned}
$$

We will also make use of the Zygmund space [31, Chapter 6, Definition 6.1]

$$
L \log L(\Omega):=\left\{u \in L^{1}(\Omega) \mid \int_{\Omega} M(u) d x<\infty\right\}, \text { where } M(u):=\chi_{\{|u|>1\}}|u| \log |u| .
$$

For $p \in[1, \infty] \backslash\{2\}$, we write $\|\cdot\|_{p}$ in place of the $\|\cdot\|_{L^{p}(\Omega)}$-norm. Throughout the paper, $\|\cdot\|$ and $(u, v)$ denote the standard $L^{2}(\Omega)$-norm and scalar product, respectively.

Finally, we make the following useful convention: For all indices $i$, the quantity $C_{i}$ denotes a non-negative constant or, alternatively, a non-negative function, which is non-decreasing in each of its arguments.

\section{Problem setting and main result}

In this section we propose a definition of weak solutions to system (1) and state our main result under the following assumptions: 


\section{Assumptions 4.1 (Initial data)}

1. $m_{0} \geqslant 0, m_{0} \not \equiv 0, m_{0} \in L \log L(\Omega)$;

2. $p_{0} \geqslant 0, p_{0} \not \equiv 0, p_{0} \in L^{\infty}(\Omega)$;

3. $0 \leqslant v_{0} \leqslant 1, v_{0} \not \equiv 0,1, v_{0}^{\frac{1}{2}} \in H^{1}(\Omega)$.

The major challenge of model (1) lies in the fact that the diffusion coefficient in equation (1a) degenerates at $v=0$. The latter seems to make it impossible to obtain an a priori estimate for the gradient of $\varphi(m)$ in some Lebesgue space for any smooth, strictly increasing function $\varphi$. As a workaround, we are forced to consider an auxiliary function which involves both $m$ and $v$ and whose gradient we are able to estimate.

This leads us to the following definition of weak solutions to (1):

Definition 4.2 (Weak solution) Let $m_{0}, p_{0}, v_{0}$ satisfy Assumptions 4.1. We call a triple of functions $m, p: \mathbb{R}_{0}^{+} \times \bar{\Omega} \rightarrow \mathbb{R}_{0}^{+}$, $v: \mathbb{R}_{0}^{+} \times \bar{\Omega} \rightarrow[0,1]$ a global weak solution of (1) if for all $0<T<\infty$ it holds that

1. $m \in L^{\infty}\left(0, T ; L^{1}(\Omega)\right)$;

2. $p \in L^{\infty}\left(0, T ; L^{\infty}(\Omega)\right), \partial_{t} p \in L^{1}\left(0, T ; L^{1}(\Omega)\right)$;

3. $v^{\frac{1}{2}} \in L^{\infty}\left(0, T ; H^{1}(\Omega)\right), \partial_{t} v \in L^{1}\left(0, T ; L^{1}(\Omega)\right)$;

4. $\nabla\left(v^{\frac{1}{2}}(m+1)^{\frac{1}{2}}\right),\left(\frac{\kappa_{m} c}{1+v c}+\frac{\kappa_{v}}{1+v}\right) v^{\frac{1}{2}}(m+1)^{\frac{1}{2}}\left(\nabla\left(v^{\frac{1}{2}}(m+1)^{\frac{1}{2}}\right)-(m+1)^{\frac{1}{2}} \nabla v^{\frac{1}{2}}\right) \in L^{1}\left(0, T ; L^{1}(\Omega)\right)$,

$\nabla\left(\int_{0}^{t} \frac{v}{1+v} m d \tau\right) \in L^{\infty}\left(0, T ; L^{2}(\Omega)\right)$;

5. ( $m, p, v)$ satisfies equation (1a) and the boundary condition (1d) in the following weak sense:

$$
\begin{aligned}
& \int_{\Omega} m_{0} \varphi d x \psi(0)-\int_{0}^{T} \int_{\Omega} m \varphi d x \psi^{\prime} d t \\
& =-\int_{0}^{T} \int_{\Omega}\left(\frac{\kappa_{m} c}{1+v c}+\frac{\kappa_{v}}{1+v}\right) 2 v^{\frac{1}{2}}(m+1)^{\frac{1}{2}}\left(\nabla\left(v^{\frac{1}{2}}(m+1)^{\frac{1}{2}}\right)-(m+1)^{\frac{1}{2}} \nabla v^{\frac{1}{2}}\right) \cdot \nabla \varphi \psi \\
& \quad+\kappa_{v} \nabla\left(\int_{0}^{t} \frac{v}{1+v} m d \tau\right) \cdot \nabla \varphi \psi^{\prime}+(-\alpha m+\beta v p) \varphi \psi d x d t
\end{aligned}
$$

for all $\varphi \in W^{1, \infty}(\Omega)$ and $\psi \in W^{1, \infty}(0, T)$ such that $\psi(T)=0$;

6. $(m, p, v)$ satisfies equations (1b)-(1c) in $L^{1}\left(0, T ; L^{1}(\Omega)\right)$;

7. $p(0)=p_{0}, v(0)=v_{0}$.

Remark 4.3 (Weak formulation) By using the chain and product rules and (where necessary) partial integration over $\Omega$ and over $[0, t]$, it can be readily checked that (2) is, indeed, a weak reformulation of (1a) and (1d). Its somewhat nonstandard form is due to the fact that $\nabla m$ in the diffusion term and the taxis flux term $\frac{\kappa_{v} m}{(1+v)^{2}} \nabla v$ might not exist even in $L_{l o c}^{1}$-sense.

Furthermore, it is clear that (2) requires e.g., $v^{\frac{1}{2}} \in L^{\infty}\left(0, T ; H^{1}(\Omega)\right)$ and not just $v \in L^{\infty}\left(0, T ; H^{1}(\Omega)\right)$. This, of course, makes a difference only near $v=0$.

Remark 4.4 (Initial conditions) Since we are looking for solutions with

$$
\begin{aligned}
& p \in W^{1,1}\left(0, T ; L^{1}(\Omega)\right), \\
& v^{\frac{1}{2}} \in H^{1}\left(0, T ; L^{1}(\Omega)\right),
\end{aligned}
$$

we have

$$
\begin{aligned}
& p \in C\left([0, T] ; L^{1}(\Omega)\right), \\
& v^{\frac{1}{2}} \in C\left([0, T] ; L^{1}(\Omega)\right) .
\end{aligned}
$$

Therefore, the initial conditions 7. in Definition 4.2 do make sense.

Our main result reads:

Theorem 4.5 (Global existence) Let $\Omega \subset \mathbb{R}^{N}, N \in \mathbb{N}$, be a smooth bounded domain and let $\alpha, \beta, \eta, \kappa_{m}, \kappa_{v}, \lambda, \mu_{p}, \mu_{v}$ be positive constants. Then, for each triple of functions $\left(m_{0}, p_{0}, v_{0}\right)$ satisfying Assumptions 4.1 there exists a global weak solution $(m, p, v)$ (in terms of Definition 4.2) to the system (1). 
The proof of Theorem 4.5 follows our approach in [26]. It is based on a suitable approximation of the degenerate system (1) by a family of systems with nondegenerate diffusion of the migrating cells, derivation of a set of a priori estimates which ensure necessary compactness and, finally, the passage to the limit. As in [26], we encounter considerable difficulties in each of the three steps due to the previously mentioned degenerate diffusion in equation (1a), due to the ODEs (1b)-(1c) having no diffusion at all (i.e., everywhere degenerate), and, finally, due to the strong couplings. However, other than in [26], equation (1a) for the moving cells lacks a logistic type remodelling term with a quadratic decay for large densities. Instead, we can only rely on the negative part of the transition term which is linear. This renders the present system even more degenerate, forcing us to define a 'weaker' weak solution in Definition 4.2 (compare also Remark 4.3 from above) and to carry out the necessary a priori estimates in an even more refined way.

Remark 4.6 (Notation for constants) We make the following useful convention: The statement that a constant depends on the parameters of the problem means that it depends on the constants $\kappa, \mu_{p}, \eta, \mu_{v}$ and $\lambda$, the norms of the initial data ( $\left.m_{0}, p_{0}, v_{0}\right)$, the space dimension $N$, and the domain $\Omega$. This dependence on the parameters is subsequently not indicated in an explicit way.

\section{Approximating problems}

In this section we introduce a family of non-degenerate approximations for problem (1). For each relaxation parameter $\varepsilon=$ $\left(\varepsilon_{1}, \varepsilon_{2}, \varepsilon_{2}\right) \in(0,1)^{3}$, the corresponding approximation reads

$$
\begin{array}{ll}
\partial_{t} m_{\varepsilon}=-\alpha m_{\varepsilon}+\beta v_{\varepsilon} p_{\varepsilon}+\varepsilon_{1} \Delta m_{\varepsilon}+\nabla \cdot\left(\frac{\kappa_{m} v_{\varepsilon} c_{\varepsilon}}{1+v_{\varepsilon} c_{\varepsilon}} \nabla m_{\varepsilon}-\frac{\kappa_{v} m_{\varepsilon}}{\left(1+v_{\varepsilon}\right)^{2}} \nabla v_{\varepsilon}\right) & \text { in } \mathbb{R}^{+} \times \Omega, \\
\partial_{t} p_{\varepsilon}=\alpha m_{\varepsilon}-\beta v_{\varepsilon} p_{\varepsilon}+\mu_{p} p_{\varepsilon}\left(1-c_{\varepsilon}-\eta v_{\varepsilon}\right) & \text { in } \mathbb{R}^{+} \times \Omega, \\
\partial_{t} v_{\varepsilon}=\mu_{v} v_{\varepsilon}\left(1-v_{\varepsilon}\right)-\lambda v_{\varepsilon} m_{\varepsilon} & \text { in } \mathbb{R}^{+} \times \Omega, \\
\varepsilon_{1} \partial_{\nu} m_{\varepsilon}+\frac{\kappa_{m} v_{\varepsilon} c_{\varepsilon}}{1+v_{\varepsilon} c_{\varepsilon}} \partial_{\nu} m_{\varepsilon}-\frac{\kappa_{v} m_{\varepsilon}}{\left(1+v_{\varepsilon}\right)^{2}} \partial_{\nu} v_{\varepsilon}=0 & \text { in } \mathbb{R}^{+} \times \partial \Omega, \\
m_{\varepsilon}(0)=m_{\varepsilon_{2} 0}, p_{\varepsilon}(0)=p_{\varepsilon_{2} 0}, v_{\varepsilon}(0)=v_{\varepsilon_{3} 0} & \text { in } \Omega,
\end{array}
$$

where

$$
c_{\varepsilon}=m_{\varepsilon}+p_{\varepsilon}
$$

and the families $\left\{m_{\varepsilon_{2} 0}\right\},\left\{p_{\varepsilon_{2} 0}\right\}$ and $\left\{v_{\varepsilon_{3} 0}\right\}$ of sufficiently smooth and nonnegative initial values are parameterized by $\varepsilon_{2}$ and $\varepsilon_{3}$, respectively. They are yet to be specified below in Subsection 5.1.

Remark 5.1 (Nonnegativity of solutions) It is easy to see that system (3) satisfies standard assumptions which ensure the nonnegativity of solutions. Indeed, if the motion terms are eliminated from the PDE, the resulting ODE system has the positivity preserving property.

For each $\varepsilon \in(0,1)^{3}$, system (3) has the form of a nondegenerate ${ }^{\dagger}$ quasilinear haptotaxis system with respect to variables $m_{\varepsilon}, p_{\varepsilon}, v_{\varepsilon}$. Thereby, the weak solutions can be defined similarly to Definition 4.2. In this case, 5. in Definition 4.2 is replaced by

$5^{\prime} .\left(m_{\varepsilon}, p_{\varepsilon}, v_{\varepsilon}\right)$ satisfies equation (3a) and the boundary condition (3d) in the following weak sense:

$$
\begin{aligned}
& \int_{\Omega} m_{\varepsilon_{2} 0} \varphi d x \psi(0)-\int_{0}^{T} \int_{\Omega} m_{\varepsilon} \varphi d x \psi^{\prime} d t \\
& =\int_{0}^{T} \int_{\Omega}-\varepsilon \nabla m_{\varepsilon} \cdot \nabla \varphi \psi d x d t \\
& \quad-\int_{0}^{T} \int_{\Omega}\left(\frac{\kappa_{m} c_{\varepsilon}}{1+v_{\varepsilon} c_{\varepsilon}}+\frac{\kappa_{v}}{1+v_{\varepsilon}}\right) 2 v_{\varepsilon}^{\frac{1}{2}}\left(m_{\varepsilon}+1\right)^{\frac{1}{2}}\left(\nabla\left(v_{\varepsilon}^{\frac{1}{2}}\left(m_{\varepsilon}+1\right)^{\frac{1}{2}}\right)-\left(m_{\varepsilon}+1\right)^{\frac{1}{2}} \nabla v_{\varepsilon}^{\frac{1}{2}}\right) \cdot \nabla \varphi \psi \\
& \quad+\kappa_{v} \nabla\left(\int_{0}^{t} \frac{v_{\varepsilon}}{1+v_{\varepsilon}} m_{\varepsilon} d \tau\right) \cdot \nabla \varphi \psi^{\prime}+\left(-\alpha m_{\varepsilon}+\beta v_{\varepsilon} p_{\varepsilon}\right) \varphi \psi d x d t
\end{aligned}
$$

for all $\varphi \in W^{1, \infty}(\Omega)$ and $\psi \in W^{1, \infty}(0, T)$ such that $\psi(T)=0$. 
The proof of the global existence of nonnegative weak solutions for the quasilinear system (3) can be carried out in a way common for semilinear haptotaxis problems. Choosing some $\theta>N+2$, one first introduces further regularising terms in the following manner:

$$
\begin{array}{ll}
\partial_{t} m_{\varepsilon}=-\alpha m_{\varepsilon}+\beta v_{\varepsilon} p_{\varepsilon}+\varepsilon_{1} \Delta m_{\varepsilon}+\nabla \cdot\left(\frac{\kappa_{m} v_{\varepsilon} c_{\varepsilon}}{1+v_{\varepsilon} c_{\varepsilon}} \nabla m_{\varepsilon}-\frac{\kappa_{v} m_{\varepsilon}}{\left(1+v_{\varepsilon}\right)^{2}} \nabla v_{\varepsilon}\right)-\varepsilon_{0} m_{\varepsilon}^{\theta} & \text { in } \mathbb{R}^{+} \times \Omega, \\
\partial_{t} p_{\varepsilon}=\alpha m_{\varepsilon}-\beta v_{\varepsilon} p_{\varepsilon}+\mu_{p} p_{\varepsilon}\left(1-c_{\varepsilon}-\eta v_{\varepsilon}\right) & \text { in } \mathbb{R}^{+} \times \Omega, \\
\partial_{t} v_{\varepsilon}=\varepsilon_{0} \Delta v_{\varepsilon}+\mu_{v} v_{\varepsilon}\left(1-v_{\varepsilon}\right)-\lambda v_{\varepsilon} m_{\varepsilon} & \text { in } \mathbb{R}^{+} \times \Omega, \\
\partial_{\nu} m_{\varepsilon}=\partial_{\nu} v_{\varepsilon}=0 & \text { in } \mathbb{R}^{+} \times \partial \Omega, \\
m_{\varepsilon}(0)=m_{\varepsilon_{2} 0}, p_{\varepsilon}(0)=p_{\varepsilon_{2} 0}, v_{\varepsilon}(0)=v_{\varepsilon_{3} 0} & \text { in } \Omega,
\end{array}
$$

where $\varepsilon_{0}>0$ is yet another small relaxation parameter. The components $p_{\varepsilon}$ and $v_{\varepsilon}$ are easily seen to be a priori bounded in $L^{\infty}$, and that holds uniformly in $\varepsilon$. As usual, the additional decay term in (5a) and the diffusion term in ( $5 \mathrm{c}$ ) are needed in order to ensure the $L^{\infty}$-boundedness of $\nabla v_{\varepsilon}$ and then of $m_{\varepsilon}$. Since the PDE-part of system (5) is nondegenerate and upper-triangular, these a priori bounds allow one to apply to (5) results from Amann's theory [32] for upper-triangular systems. They ensure the existence of a unique classical solution of (5). Finally, uniform w.r.t. $\varepsilon_{0}$ estimates, a compactness argument, and a limit procedure as $\varepsilon_{0} \rightarrow 0$ lead to a solution to system (3). We omit further details here since they follow a standard route and because we will derive a priori estimates and compactness in suitable spaces in a more general situation of system (3), i.e., even without additional regularising terms, in Section 6 below.

It is clear that for $\varepsilon=0$ we regain - at least formally - the original degenerate haptotaxis system (1). As it turns out (see the subsequent Section 7), a weak solution to (1) can be obtained as a limit of a sequence of solutions to (3).

In order to shorten the writing, we will sometimes use the following notation for the flux and reaction terms, respectively:

$$
\begin{aligned}
q_{\varepsilon} & :=\varepsilon_{1} \nabla m_{\varepsilon}+\frac{\kappa_{m} v_{\varepsilon} c_{\varepsilon}}{1+v_{\varepsilon} c_{\varepsilon}} \nabla m_{\varepsilon}-\frac{\kappa_{v} m_{\varepsilon}}{\left(1+v_{\varepsilon}\right)^{2}} \nabla v_{\varepsilon}, \\
f_{\varepsilon} & :=-\alpha m_{\varepsilon}+\beta v_{\varepsilon} p_{\varepsilon}
\end{aligned}
$$

\subsection{Approximating initial data}

Our next step is to construct a suitable family of approximations to the initial data. Since we assume that $\left(m_{0}, p_{0}, v_{0}\right)$ satisfies Assumptions 4.1, there exists for each $\left(\varepsilon_{2}, \varepsilon_{3}\right) \in(0,1)^{2}$ an approximation triple $\left(m_{\varepsilon_{2} 0}, p_{\varepsilon_{2} 0}, v_{\varepsilon_{3} 0}\right)$ with the following properties:

$$
\begin{aligned}
& m_{\varepsilon_{2} 0}, p_{\varepsilon_{2} 0}, v_{\varepsilon_{3} 0}^{\frac{1}{2}} \in W^{1, \infty}(\Omega), \\
& m_{\varepsilon_{2} 0}, p_{\varepsilon_{2} 0} \geqslant 0,0 \leqslant v_{\varepsilon_{3} 0} \leqslant 1 \text { in } \bar{\Omega}, m_{\varepsilon_{2} 0}, p_{\varepsilon_{2} 0}, v_{\varepsilon_{3} 0} \not \equiv 0, \\
& \left\|M\left(m_{\varepsilon_{2} 0}\right)\right\|_{L^{1}(\Omega)} \leqslant 2\left\|M\left(m_{0}\right)\right\|_{L^{1}(\Omega)}, \\
& \left\|\nabla v_{\varepsilon_{3} 0}^{\frac{1}{2}}\right\| \leqslant 2\left\|v_{0}^{\frac{1}{2}}\right\|_{H^{1}(\Omega)}, \\
& \left\|m_{\varepsilon_{2} 0}-m_{0}\right\|_{L^{1}(\Omega)} \leqslant \varepsilon_{2}, \\
& \left\|p_{\varepsilon_{2} 0}-p_{0}\right\|_{L^{\infty}(\Omega)} \leqslant \varepsilon_{2}, \\
& \left\|v_{\varepsilon_{3} 0}^{\frac{1}{2}}-v_{0}^{\frac{1}{2}}\right\| \leqslant \varepsilon_{3} .
\end{aligned}
$$

Recall that our aim is to pass to the limit for $\varepsilon \rightarrow 0$ in the approximating problem. Since equation (3c) is an ODE, the set $\{v(t, \cdot)=0\}$ is preserved in time (possibly up to some subsets of measure zero). Therefore, it turns out that we have to pay particular care at the set $\left\{v_{\varepsilon_{3} 0}=0\right\}$ whose interior should not shrink substantially with respect to $\left\{v_{0}=0\right\}$. Following the idea from [26], we assume that

$$
\left|\left\{v_{0}=0\right\} \backslash \operatorname{int}\left\{v_{\varepsilon_{3} 0}=0\right\}\right| \leqslant \varepsilon_{3} .
$$

Indeed, to justify (14) we recall here our argument from [26] for the convenience of the reader. Due to a Lusin property for Sobolev functions [33, Chapter 6, Theorem 6.14], there exists a function $\xi$ such that

$$
\begin{aligned}
& \xi \in W^{1, \infty}(\Omega), \\
& \|\xi\|_{H^{1}(\Omega)} \leqslant 2\left\|v_{0}^{\frac{1}{2}}\right\|_{H^{1}(\Omega)}, \\
& \left|\left\{\xi \neq v_{0}^{\frac{1}{2}}\right\}\right| \leqslant \frac{\varepsilon_{3}}{4} .
\end{aligned}
$$

We define

$$
v_{\varepsilon_{3} 0}:=\left(\min \left\{\xi_{+}, 1\right\}-\frac{\varepsilon_{3}}{2|\Omega|}\right)_{+}^{2}
$$


Let us check that $v_{\varepsilon_{3} 0}$ satisfies the above assumptions. Indeed, due to (16)-(17), we have that

$$
\begin{aligned}
& v_{\varepsilon_{3} 0}^{\frac{1}{2}} \in W^{1, \infty}(\Omega), \\
& \left\|\nabla v_{\varepsilon_{3} 0}^{\frac{1}{2}}\right\| \leqslant\|\nabla \xi\| \leqslant 2\left\|v_{0}^{\frac{1}{2}}\right\|_{H^{1}(\Omega)},
\end{aligned}
$$

and

$$
\begin{aligned}
\left\|v_{\varepsilon_{3} 0}^{\frac{1}{2}}-v_{0}^{\frac{1}{2}}\right\| & \leqslant 2\left|\left\{\xi \neq v_{0}^{\frac{1}{2}}\right\}\right|+\left\|\chi_{\left\{\xi=v_{0}^{\frac{1}{2}}\right\}}\left(\left(\xi-\frac{\varepsilon_{3}}{2|\Omega|}\right)_{+}-\xi\right)\right\| \\
& \leqslant \varepsilon_{3} .
\end{aligned}
$$

Moreover, it holds that

$$
\{\xi=0\} \subset\left\{\min \left\{\xi_{+}, 1\right\}<\frac{\varepsilon_{3}}{2|\Omega|}\right\} \subset \operatorname{int}\left\{\min \left\{\xi_{+}, 1\right\} \leqslant \frac{\varepsilon_{3}}{2|\Omega|}\right\} \cup \partial \Omega=\operatorname{int}\left\{v_{\varepsilon_{3} 0}=0\right\} \cup \partial \Omega .
$$

Combining (18) and (19), we obtain (15).

\section{A priori estimates}

In this section we establish, based on system (3), several uniform a priori estimates for the functions $m_{\varepsilon}, p_{\varepsilon}, v_{\varepsilon}$ and their combinations, which we will use in the existence proof (see Section 7 below). Our calculations make use of the regularity which the solutions of (3) do have. While operating with the weak derivatives, we use the weak chain and product rules. Another way to justify the calculation is via further approximations, as was done in [26].

Uniform boundedness of $v_{\varepsilon}$

Since the ODE (3c) has the form

$$
\partial_{t} v_{\varepsilon}=f_{v}\left(v_{\varepsilon}, m_{\varepsilon}\right)
$$

with $f_{v}(1, m) \leqslant 0$ for all $m \geqslant 0$, and the initial value satisfies $v_{\varepsilon_{3} 0} \leqslant 1$ (compare (9)), we obtain using standard ODE theory that

$$
v_{\varepsilon} \leqslant 1 \text { in }(0, T) \times \Omega .
$$

holds a priori. Below we will use this simple estimate without referring to it explicitly.

Uniform boundedness of $p_{\varepsilon}$

Equation (3b) for $p_{\varepsilon}$ can be rewritten in the following way:

$$
\partial_{t} p_{\varepsilon}=-\left(\mu_{p} p_{\varepsilon}-\alpha\right) m_{\varepsilon}-\left(\beta+\mu_{p} \eta\right) v_{\varepsilon} p_{\varepsilon}+\mu_{p} p_{\varepsilon}\left(1-p_{\varepsilon}\right) .
$$

Since $m_{\varepsilon}, p_{\epsilon}, v_{\varepsilon} \geqslant 0$, we obtain using standard ODE theory that

$$
p_{\varepsilon} \leqslant \max \left\{1, \frac{\alpha}{\mu_{p}},\left\|p_{\varepsilon}(0)\right\|_{L^{\infty}(\Omega)}\right\} .
$$

Combining (13) and (20), we obtain that

$$
p_{\varepsilon} \leqslant C_{p}
$$

\section{Energy-type estimates}

We now turn to equation (3c) for $v_{\varepsilon}$. Unlike the model in [34], the haptotaxis coefficient lacks a factor $v$ in the denominator, whose presence was essential for obtaining estimates involving $\nabla v$ (and which relied on differentiating the equation for $v$ ). Here we compensate the absence of $v$ by rearranging equation (3c) in a convenient way. On both sides of the equation we divide by $v_{\varepsilon}^{\frac{1}{2}}\left(1+v_{\varepsilon}\right)$ and then apply the gradient operator. Thus we obtain that

$$
\partial_{t} \nabla \int_{0}^{v_{\varepsilon}} \frac{1}{s^{\frac{1}{2}}(1+s)} d s=-\lambda \frac{v_{\varepsilon}^{\frac{1}{2}}}{1+v_{\varepsilon}} \nabla m_{\varepsilon}-\frac{\lambda\left(1-v_{\varepsilon}\right) m_{\varepsilon}+\mu_{v}\left(-1+4 v_{\varepsilon}+v_{\varepsilon}^{2}\right)}{\left(1+v_{\varepsilon}\right)^{2}} \nabla v_{\varepsilon}^{\frac{1}{2}}
$$


Further, we multiply (3a) by $\ln m_{\varepsilon}$ and (22) by $\frac{\kappa_{v}}{\lambda} \nabla \int_{0}^{v_{\varepsilon}} \frac{1}{s^{\frac{1}{2}}(1+s)} d s$ and integrate over $\Omega$ using partial integration and the boundary conditions where necessary. Adding the resulting identities together, we obtain after some calculation that

$$
\begin{aligned}
& \frac{d}{d t}\left(\left(1, m_{\varepsilon} \ln m_{\varepsilon}-m_{\varepsilon}\right)+\frac{2 \kappa_{v}}{\lambda}\left(\frac{1}{\left(1+v_{\varepsilon}\right)^{2}},\left|\nabla v_{\varepsilon}^{\frac{1}{2}}\right|^{2}\right)\right)+\varepsilon_{1}\left\|\nabla m_{\varepsilon}^{\frac{1}{2}}\right\|^{2}+4\left(\frac{\kappa_{m} v_{\varepsilon} c_{\varepsilon}}{1+v_{\varepsilon} c_{\varepsilon}},\left|\nabla m_{\varepsilon}^{\frac{1}{2}}\right|^{2}\right) \\
& +\frac{2 \kappa_{v}}{\lambda}\left(\lambda\left(1-v_{\varepsilon}\right) m_{\varepsilon}+5 \mu_{v} v_{\varepsilon}+\mu_{v} v_{\varepsilon}^{2}, \frac{\left|\nabla v_{\varepsilon}^{\frac{1}{2}}\right|^{2}}{\left(1+v_{\varepsilon}\right)^{3}}\right)+\alpha\left(m_{\varepsilon}, \ln m_{\varepsilon}\right) \\
\leqslant & \beta\left(v_{\varepsilon} p_{\varepsilon}, \ln m_{\varepsilon}\right)+\frac{2 \mu_{v} \kappa_{v}}{\lambda}\left(\frac{1}{\left(1+v_{\varepsilon}\right)^{2}},\left|\nabla v_{\varepsilon}^{\frac{1}{2}}\right|^{2}\right)
\end{aligned}
$$

By using the Gronwall lemma and (21), we thus arrive, for arbitrary $T \in \mathbb{R}^{+}$, at the estimates

$$
\begin{aligned}
& \sup _{t \in[0, T]}\left(\chi_{\left\{m_{\varepsilon}>1\right\}}, m_{\varepsilon} \ln m_{\varepsilon}\right) \leqslant C_{1}(T), \\
& \sup _{t \in[0, T]}\left\|\nabla v_{\varepsilon}^{\frac{1}{2}}\right\|^{2} \leqslant C_{1}(T), \\
& \int_{0}^{T}\left(\frac{v_{\varepsilon} c_{\varepsilon}}{1+v_{\varepsilon} c_{\varepsilon}},\left|\nabla m_{\varepsilon}^{\frac{1}{2}}\right|^{2}\right) d t \leqslant C_{1}(T), \\
& \int_{0}^{T}\left(\left(1-v_{\varepsilon}\right) m_{\varepsilon},\left|\nabla v_{\varepsilon}^{\frac{1}{2}}\right|^{2}\right) d t \leqslant C_{1}(T), \\
& \int_{0}^{T}\left(v_{\varepsilon} p_{\varepsilon},-\chi_{\left\{m_{\varepsilon}<1\right\}} \ln m_{\varepsilon}\right) d t \leqslant C_{1}(T) \\
& \int_{0}^{T}\left\|\nabla m_{\varepsilon}^{\frac{1}{2}}\right\|^{2} d t \leqslant \varepsilon_{1}^{-1} C_{1}(T) .
\end{aligned}
$$

Since $s \mapsto \frac{s}{1+s}$ is a monotonically increasing function, (25) yields that

$$
\begin{aligned}
\int_{0}^{T}\left(\frac{v_{\varepsilon}}{1+v_{\varepsilon} m_{\varepsilon}},\left|\nabla m_{\varepsilon}\right|^{2}\right) d t & =4 \int_{0}^{T}\left(\frac{v_{\varepsilon} m_{\varepsilon}}{1+v_{\varepsilon} m_{\varepsilon}},\left|\nabla m_{\varepsilon}^{\frac{1}{2}}\right|^{2}\right) d t \\
& \leqslant 4 \int_{0}^{T}\left(\frac{v_{\varepsilon} c_{\varepsilon}}{1+v_{\varepsilon} c_{\varepsilon}},\left|\nabla m_{\varepsilon}^{\frac{1}{2}}\right|^{2}\right) d t \\
& \leqslant C_{2}(T) .
\end{aligned}
$$

Consequently, we also have that

$$
\begin{aligned}
\int_{0}^{T}\left(v_{\varepsilon},\left|\nabla\left(1+m_{\varepsilon}\right)^{\frac{1}{2}}\right|^{2}\right) d t & =\frac{1}{4} \int_{0}^{T}\left(\frac{v_{\varepsilon}}{1+m_{\varepsilon}},\left|\nabla m_{\varepsilon}\right|^{2}\right) d t \\
& \leqslant \frac{1}{4} \int_{0}^{T}\left(\frac{v_{\varepsilon}}{1+v_{\varepsilon} m_{\varepsilon}},\left|\nabla m_{\varepsilon}\right|^{2}\right) d t \\
& \leqslant C_{3}(T)
\end{aligned}
$$

Uniform integrability of $m_{\varepsilon}$

It follows with (23) that

$$
\left\|m_{\varepsilon}\right\|_{L^{\infty}\left(0, T ; L^{1}(\Omega)\right)} \leqslant C_{4}(T) .
$$

Moreover, due to the de la Vallée-Poussin theorem, we conclude with (23) that

$$
\left\{m_{\varepsilon}\right\} \text { is uniformly integrable in }(0, T) \times \Omega \text {. }
$$

Uniform integrability of $\nabla\left(v_{\varepsilon}^{\frac{1}{2}}\left(m_{\varepsilon}+1\right)^{\frac{1}{2}}\right)$

Due to (32), it holds that

$$
\left\{v_{\varepsilon}^{\frac{1}{2}}\left(m_{\varepsilon}+1\right)^{\frac{1}{2}}\right\} \text { is uniformly integrable in }(0, T) \times \Omega \text {. }
$$


We compute that

$$
\nabla\left(v_{\varepsilon}^{\frac{1}{2}}\left(m_{\varepsilon}+1\right)^{\frac{1}{2}}\right)=v_{\varepsilon}^{\frac{1}{2}} \nabla\left(m_{\varepsilon}+1\right)^{\frac{1}{2}}+\left(m_{\varepsilon}+1\right)^{\frac{1}{2}} \nabla v_{\varepsilon}^{\frac{1}{2}} .
$$

Combining (24), (30), (32), (34) and using the de la Vallée-Poussin theorem and Lemma A.1, we conclude that

$$
\left\{\nabla\left(v_{\varepsilon}^{\frac{1}{2}}\left(m_{\varepsilon}+1\right)^{\frac{1}{2}}\right)\right\} \text { is uniformly integrable in }(0, T) \times \Omega \text {. }
$$

Uniform integrability of the reaction term in (3a)

It immediately follows with (21), (31), (32) that

$$
\left\{f_{\varepsilon}\right\} \text { is uniformly integrable in }(0, T) \times \Omega
$$

and

$$
\left\|f_{\varepsilon}\right\|_{L^{\infty}\left(0, T ; L^{1}(\Omega)\right)} \leqslant C_{5}(T) .
$$

Uniform integrability of the diffusion flux in (3a)

We first deal with the relaxation term. We have that

$$
\varepsilon_{1}^{\frac{1}{2}}\left|\nabla m_{\varepsilon}\right|=2 \varepsilon_{1}^{\frac{1}{2}}\left|\nabla m_{\varepsilon}^{\frac{1}{2}}\right| m_{\varepsilon}^{\frac{1}{2}},
$$

Using the Hölder inequality, we obtain with (28), (31) and (38) that

$$
\varepsilon_{1}\left\|\nabla m_{\varepsilon}\right\|_{L^{1}\left(0, T ; L^{1}(\Omega)\right)} \leqslant \varepsilon_{1}^{\frac{1}{2}} C_{6}(T) .
$$

For the degenerate part of the diffusion flux, we have that

$$
\frac{v_{\varepsilon} c_{\varepsilon}\left|\nabla m_{\varepsilon}\right|}{1+v_{\varepsilon} c_{\varepsilon}}=2\left(\frac{v_{\varepsilon} c_{\varepsilon}}{1+v_{\varepsilon} c_{\varepsilon}}\right)^{\frac{1}{2}} m_{\varepsilon}^{\frac{1}{2}}\left(\frac{v_{\varepsilon} c_{\varepsilon}}{1+v_{\varepsilon} c_{\varepsilon}}\left|\nabla m_{\varepsilon}^{\frac{1}{2}}\right|^{2}\right)^{\frac{1}{2}} \text {. }
$$

Combining (25) and (32), we obtain with Lemma A.1 that

$$
\left\{\frac{v_{\varepsilon} c_{\varepsilon} \nabla m_{\varepsilon}}{1+v_{\varepsilon} c_{\varepsilon}}\right\} \text { is uniformly integrable in }(0, T) \times \Omega
$$

so that

$$
\left\|\frac{v_{\varepsilon} c_{\varepsilon}\left|\nabla m_{\varepsilon}\right|}{1+v_{\varepsilon} c_{\varepsilon}}\right\|_{L^{1}\left(0, T ; L^{1}(\Omega)\right)} \leqslant C_{7}(T)
$$

Uniform integrability of the taxis flux in (3a)

Let us next consider the taxis part of the flux. We compute that

$$
\begin{aligned}
\frac{m_{\varepsilon}}{\left(1+v_{\varepsilon}\right)^{2}} \nabla v_{\varepsilon} & =m_{\varepsilon} \nabla \frac{v_{\varepsilon}}{1+v_{\varepsilon}} \\
& =\nabla\left(\frac{v_{\varepsilon} m_{\varepsilon}}{1+v_{\varepsilon}}\right)-\frac{v_{\varepsilon}}{1+v_{\varepsilon}} \nabla m_{\varepsilon} .
\end{aligned}
$$

For the second summand on the right-hand side of (43), we have that

$$
\frac{v_{\varepsilon}}{1+v_{\varepsilon}} \nabla m_{\varepsilon}=2 \frac{v_{\varepsilon}^{\frac{1}{2}}}{1+v_{\varepsilon}}\left(m_{\varepsilon}+1\right)^{\frac{1}{2}} v_{\varepsilon}^{\frac{1}{2}} \nabla\left(m_{\varepsilon}+1\right)^{\frac{1}{2}}
$$

We use (30), (32) and Lemma A.1 in order to conclude from (44) that

$$
\left\{\frac{v_{\varepsilon}}{1+v_{\varepsilon}} \nabla m_{\varepsilon}\right\} \text { is uniformly integrable in }(0, T) \times \Omega \text {. }
$$

As for the first summand on the right-hand side of (43), we seek for an estimate for its integral over $(0, t)$ (compare Definition 4.2). On both sides of equation (3c), we divide by $1+v_{\varepsilon}$, apply the space gradient and finally integrate over $(0, t)$. This yields that

$$
\frac{1}{1+v_{\varepsilon}} \nabla v_{\varepsilon}(t)-\frac{1}{1+v_{\varepsilon_{3} 0}} \nabla v_{\varepsilon_{3} 0}=\left.\mu_{v} \int_{0}^{t}\left(\frac{s(1-s)}{1+s}\right)^{\prime}\right|_{s=v_{\varepsilon}} \nabla v_{\varepsilon} d \tau-\lambda \nabla\left(\int_{0}^{t} \frac{v_{\varepsilon} m_{\varepsilon}}{1+v_{\varepsilon}} d \tau\right) .
$$

Since $s \mapsto \frac{s(1-s)}{1+s}$ is continuously differentiable, we conclude from (46) using (24) that

$$
\left\|\nabla\left(\int_{0}^{t} \frac{v_{\varepsilon} m_{\varepsilon}}{1+v_{\varepsilon}} d \tau\right)\right\|_{L^{\infty}\left(0, T ; L^{2}(\Omega)\right)} \leqslant C_{8}(T)
$$


Estimates involving $\partial_{t} v_{\varepsilon}$

We divide equation $(3 c)$ by $v_{\varepsilon}$ :

$$
\frac{1}{v_{\varepsilon}} \partial_{t} v_{\varepsilon}=\mu_{v}\left(1-v_{\varepsilon}\right)-\lambda m_{\varepsilon} .
$$

Together with (31), (48) yields that

$$
\left\|\frac{1}{v_{\varepsilon}} \partial_{t} v_{\varepsilon}\right\|_{L^{\infty}\left(0, T ; L^{1}(\Omega)\right)} \leqslant C_{9}(T),
$$

so that, consequently,

$$
\left\|\partial_{t} v_{\varepsilon}^{\frac{1}{2}}\right\|_{L^{\infty}\left(0, T ; L^{1}(\Omega)\right)} \leqslant C_{10}(T) .
$$

Estimates for $\ln \left(1+v_{\varepsilon}^{\frac{1}{2}} m_{\varepsilon}\right)$

Above we obtained uniform (in $\varepsilon$ ) estimates for both time and spacial derivatives of $v_{\varepsilon}$. Owing to the fact that the original diffusion coefficient in (1a) is degenerate in $v$, it does not seem possible to obtain similar estimates for $m_{\varepsilon}$ or, at least, for $\varphi\left(m_{\varepsilon}\right)$ for a smooth, strictly increasing, and independent of $\varepsilon$ function $\varphi$. In order to overcome this difficulty and gain some information on $m_{\varepsilon}$ in the set $\left\{v_{\varepsilon}>0\right\}$, we introduce for $\varepsilon \in(0,1)$ an auxiliary function which involves both $m_{\varepsilon}$ and $v_{\varepsilon}$ :

$$
u_{\varepsilon}:=\ln \left(1+v_{\varepsilon}^{\frac{1}{2}} m_{\varepsilon}\right)
$$

Since

$$
0 \leqslant \ln \left(1+v_{\varepsilon}^{\frac{1}{2}} m_{\varepsilon}\right) \leqslant m_{\varepsilon}
$$

we obtain with (32) that

$$
\left\{u_{\varepsilon}\right\} \text { is uniformly integrable in }(0, T) \times \Omega \text {. }
$$

As it turns out, the family $\left\{u_{\varepsilon}\right\}$ is (strongly) precompact in $L^{1}\left(0, T ; L^{1}(\Omega)\right)$. To prove this, we need uniform estimates for the partial derivatives of $u_{\varepsilon}$ in some parabolic Sobolev spaces.

We first study the spatial gradient of $u_{\varepsilon}$. We compute that

$$
\nabla u_{\varepsilon}=\frac{m_{\varepsilon}}{1+v_{\varepsilon}^{\frac{1}{2}} m_{\varepsilon}} \nabla v_{\varepsilon}^{\frac{1}{2}}+\frac{v_{\varepsilon}^{\frac{1}{2}}}{1+v_{\varepsilon}^{\frac{1}{2}} m_{\varepsilon}} \nabla m_{\varepsilon} .
$$

Using the trivial inequality

$$
1 \leqslant v_{\varepsilon}^{\frac{1}{2}}+\left(1-v_{\varepsilon}\right)^{\frac{1}{2}}
$$

we estimate the first summand on the right-hand side of (52) in the following way:

$$
\begin{aligned}
\frac{m_{\varepsilon}\left|\nabla v_{\varepsilon}^{\frac{1}{2}}\right|}{1+v_{\varepsilon}^{\frac{1}{2}} m_{\varepsilon}} & \leqslant \frac{v_{\varepsilon}^{\frac{1}{2}} m_{\varepsilon}\left|\nabla v_{\varepsilon}^{\frac{1}{2}}\right|}{1+v_{\varepsilon}^{\frac{1}{2}} m_{\varepsilon}}+\frac{\left(1-v_{\varepsilon}\right)^{\frac{1}{2}} m_{\varepsilon}\left|\nabla v_{\varepsilon}^{\frac{1}{2}}\right|}{1+v_{\varepsilon}^{\frac{1}{2}} m_{\varepsilon}} \\
& \leqslant\left|\nabla v_{\varepsilon}^{\frac{1}{2}}\right|+m_{\varepsilon}^{\frac{1}{2}}\left(\left(1-v_{\varepsilon}\right) m_{\varepsilon}\left|\nabla v_{\varepsilon}^{\frac{1}{2}}\right|^{2}\right)^{\frac{1}{2}} \\
& \leqslant\left|\nabla v_{\varepsilon}^{\frac{1}{2}}\right|+\frac{1}{2} m_{\varepsilon}+\frac{1}{2}\left(1-v_{\varepsilon}\right) m_{\varepsilon}\left|\nabla v_{\varepsilon}^{\frac{1}{2}}\right|^{2} .
\end{aligned}
$$

Using estimates (24), (26), (31), we conclude from (54) that

$$
\left\|\frac{m_{\varepsilon}\left|\nabla v_{\varepsilon}^{\frac{1}{2}}\right|}{1+v_{\varepsilon}^{\frac{1}{2}} m_{\varepsilon}}\right\|_{L^{1}\left(0, T ; L^{1}(\Omega)\right)} \leqslant C_{11}(T) .
$$


For the second summand on the right-hand side of (52), we have that

$$
\frac{v_{\varepsilon}^{\frac{1}{2}}\left|\nabla m_{\varepsilon}\right|}{1+v_{\varepsilon}^{\frac{1}{2}} m_{\varepsilon}} \leqslant \frac{v_{\varepsilon}^{\frac{1}{2}}\left|\nabla m_{\varepsilon}\right|}{1+v_{\varepsilon} m_{\varepsilon}} \leqslant \frac{v_{\varepsilon}^{\frac{1}{2}}\left|\nabla m_{\varepsilon}\right|}{\left(1+v_{\varepsilon} m_{\varepsilon}\right)^{\frac{1}{2}}}
$$

Due to (29), (56) yields that

$$
\left\|\frac{v_{\varepsilon}^{\frac{1}{2}}\left|\nabla m_{\varepsilon}\right|}{1+v_{\varepsilon}^{\frac{1}{2}} m_{\varepsilon}}\right\|_{L^{2}((0, T) \times \Omega)} \leqslant C_{12}(T) .
$$

Altogether, we obtain from (52) with (55), (57) that

$$
\left\|\nabla u_{\varepsilon}\right\|_{L^{1}\left(0, T ; L^{1}(\Omega)\right)} \leqslant C_{13}(T) .
$$

Next, we deal with the time derivative of $u_{\varepsilon}$. We compute that

$$
\partial_{t} u_{\varepsilon}=\frac{1}{2} \frac{v_{\varepsilon}^{\frac{1}{2}} m_{\varepsilon}}{1+v_{\varepsilon}^{\frac{1}{2}} m_{\varepsilon}} \frac{1}{v_{\varepsilon}} \partial_{t} v_{\varepsilon}+\frac{v_{\varepsilon}^{\frac{1}{2}}}{1+v_{\varepsilon}^{\frac{1}{2}} m_{\varepsilon}} \partial_{t} m_{\varepsilon}
$$

We estimate the first summand on the right-hand side of (59) as follows:

$$
\frac{1}{2}\left|\frac{v_{\varepsilon}^{\frac{1}{2}} m_{\varepsilon}}{1+v_{\varepsilon}^{\frac{1}{2}} m_{\varepsilon}} \frac{1}{v_{\varepsilon}} \partial_{t} v_{\varepsilon}\right| \leqslant \frac{1}{2}\left|\frac{1}{v_{\varepsilon}} \partial_{t} v_{\varepsilon}\right|
$$

Combining (49) and (60), we obtain that

$$
\frac{1}{2}\left\|\frac{v_{\varepsilon}^{\frac{1}{2}} m_{\varepsilon}}{1+v_{\varepsilon}^{\frac{1}{2}} m_{\varepsilon}} \frac{1}{v_{\varepsilon}} \partial_{t} v_{\varepsilon}\right\|_{L^{\infty}\left(0, T ; L^{1}(\Omega)\right)} \leqslant C_{14} .
$$

In order to estimate the second summand on the right-hand side of (59), we multiply both sides of equation (3a) by $\frac{v_{\varepsilon}^{\frac{1}{2}}}{1+v_{\varepsilon}^{\frac{1}{2}} m_{\varepsilon}}$ and obtain (compare the notation in (6)-(7)) that

$$
\frac{v_{\varepsilon}^{\frac{1}{2}}}{1+v_{\varepsilon}^{\frac{1}{2}} m_{\varepsilon}} \partial_{t} m_{\varepsilon}=\nabla \cdot\left(\frac{v_{\varepsilon}^{\frac{1}{2}}}{1+v_{\varepsilon}^{\frac{1}{2}} m_{\varepsilon}} q_{\varepsilon}\right)-q_{\varepsilon} \cdot \nabla \frac{v_{\varepsilon}^{\frac{1}{2}}}{1+v_{\varepsilon}^{\frac{1}{2}} m_{\varepsilon}}+\frac{v_{\varepsilon}^{\frac{1}{2}}}{1+v_{\varepsilon}^{\frac{1}{2}} m_{\varepsilon}} f_{\varepsilon} .
$$

Since

$$
\frac{v_{\varepsilon}^{\frac{1}{2}}}{1+v_{\varepsilon}^{\frac{1}{2}} m_{\varepsilon}} \leqslant 1
$$

it holds that

$$
\frac{v_{\varepsilon}^{\frac{1}{2}}}{1+v_{\varepsilon}^{\frac{1}{2}} m_{\varepsilon}}\left|f_{\varepsilon}\right| \leqslant\left|f_{\varepsilon}\right| .
$$

Hence, we conclude with (37) that

$$
\left\|\frac{v_{\varepsilon}^{\frac{1}{2}}}{1+v_{\varepsilon}^{\frac{1}{2}} m_{\varepsilon}} f_{\varepsilon}\right\|_{L^{\infty}\left(0, T ; L^{1}(\Omega)\right)} \leqslant C_{15}(T) .
$$

For the term inside the divergence operator in (62), we have that

$$
\begin{aligned}
\frac{v_{\varepsilon}^{\frac{1}{2}}}{1+v_{\varepsilon}^{\frac{1}{2}} m_{\varepsilon}}\left|q_{\varepsilon}\right| & \leqslant \frac{v_{\varepsilon}^{\frac{1}{2}}}{1+v_{\varepsilon}^{\frac{1}{2}} m_{\varepsilon}}\left(\varepsilon_{1}\left|\nabla m_{\varepsilon}\right|+\kappa_{m} \frac{v_{\varepsilon} c_{\varepsilon}\left|\nabla m_{\varepsilon}\right|}{1+v_{\varepsilon} c_{\varepsilon}}+\kappa_{v} \frac{2 v_{\varepsilon}^{\frac{1}{2}} m_{\varepsilon}\left|\nabla v_{\varepsilon}^{\frac{1}{2}}\right|}{\left(1+v_{\varepsilon}\right)^{2}}\right) \\
& \leqslant \varepsilon_{1}\left|\nabla m_{\varepsilon}\right|+\kappa_{m} \frac{v_{\varepsilon} c_{\varepsilon}\left|\nabla m_{\varepsilon}\right|}{1+v_{\varepsilon} c_{\varepsilon}}+2 \kappa_{v}\left|\nabla v_{\varepsilon}^{\frac{1}{2}}\right| .
\end{aligned}
$$


Using (24), (39), (42), we obtain from (64) that

$$
\left\|\frac{v_{\varepsilon}^{\frac{1}{2}}}{1+v_{\varepsilon}^{\frac{1}{2}} m_{\varepsilon}} q_{\varepsilon}\right\|_{L^{1}\left(0, T ; L^{1}(\Omega)\right)} \leqslant C_{16}(T) .
$$

It remains to estimate the second term on the right-hand side of (62). We compute that

$$
\nabla \frac{v_{\varepsilon}^{\frac{1}{2}}}{1+v_{\varepsilon}^{\frac{1}{2}} m_{\varepsilon}}=-\frac{v_{\varepsilon}}{\left(1+v_{\varepsilon}^{\frac{1}{2}} m_{\varepsilon}\right)^{2}} \nabla m_{\varepsilon}+\frac{1}{\left(1+v_{\varepsilon}^{\frac{1}{2}} m_{\varepsilon}\right)^{2}} \nabla v_{\varepsilon}^{\frac{1}{2}},
$$

so that

$$
\begin{aligned}
\left|q_{\varepsilon} \cdot \nabla \frac{v_{\varepsilon}^{\frac{1}{2}}}{1+v_{\varepsilon}^{\frac{1}{2}} m_{\varepsilon}}\right| & \leqslant\left|q_{\varepsilon}\right|\left|\nabla \frac{v_{\varepsilon}^{\frac{1}{2}}}{1+v_{\varepsilon}^{\frac{1}{2}} m_{\varepsilon}}\right| \\
& \leqslant\left(\varepsilon_{1}\left|\nabla m_{\varepsilon}\right|+\frac{\kappa_{m} v_{\varepsilon} c_{\varepsilon}\left|\nabla m_{\varepsilon}\right|}{1+v_{\varepsilon} c_{\varepsilon}}+\frac{2 v_{\varepsilon}^{\frac{1}{2}} m_{\varepsilon}\left|\nabla v_{\varepsilon}^{\frac{1}{2}}\right|}{\left(1+v_{\varepsilon}\right)^{2}}\right)\left(\frac{v_{\varepsilon}\left|\nabla m_{\varepsilon}\right|}{\left(1+v_{\varepsilon}^{\frac{1}{2}} m_{\varepsilon}\right)^{2}}+\frac{\left|\nabla v_{\varepsilon}^{\frac{1}{2}}\right|}{\left(1+v_{\varepsilon}^{\frac{1}{2}} m_{\varepsilon}\right)^{2}}\right) \\
& \leqslant C_{17}\left(\varepsilon_{1}\left|\nabla m_{\varepsilon}\right|+\frac{v_{\varepsilon} c_{\varepsilon}\left|\nabla m_{\varepsilon}\right|}{1+v_{\varepsilon} c_{\varepsilon}}+v_{\varepsilon}^{\frac{1}{2}} m_{\varepsilon}\left|\nabla v_{\varepsilon}^{\frac{1}{2}}\right|\right)\left(\frac{\left|\nabla v_{\varepsilon}^{\frac{1}{2}}\right|}{\left(1+v_{\varepsilon}^{\frac{1}{2}} m_{\varepsilon}\right)^{2}}+\frac{\left|\nabla m_{\varepsilon}\right|}{\left(1+v_{\varepsilon}^{\frac{1}{2}} m_{\varepsilon}\right)^{2}}\right) .
\end{aligned}
$$

Using (53) and (21) where necessary, we get the following estimates:

$$
\begin{aligned}
& \left|\nabla m_{\varepsilon}\right| \frac{v_{\varepsilon}\left|\nabla m_{\varepsilon}\right|}{\left(1+v_{\varepsilon}^{\frac{1}{2}} m_{\varepsilon}\right)^{2}} \leqslant \frac{v_{\varepsilon}\left|\nabla m_{\varepsilon}\right|^{2}}{1+v_{\varepsilon} m_{\varepsilon}} \\
& \frac{v_{\varepsilon} c_{\varepsilon}\left|\nabla m_{\varepsilon}\right|}{1+v_{\varepsilon} c_{\varepsilon}} \frac{v_{\varepsilon}\left|\nabla m_{\varepsilon}\right|}{\left(1+v_{\varepsilon}^{\frac{1}{2}} m_{\varepsilon}\right)^{2}} \leqslant \frac{v_{\varepsilon}\left|\nabla m_{\varepsilon}\right|^{2}}{1+v_{\varepsilon} m_{\varepsilon}} \\
& v_{\varepsilon}^{\frac{1}{2}} m_{\varepsilon}\left|\nabla v_{\varepsilon}^{\frac{1}{2}}\right| \frac{v_{\varepsilon}\left|\nabla m_{\varepsilon}\right|}{\left(1+v_{\varepsilon}^{\frac{1}{2}} m_{\varepsilon}\right)^{2}} \leqslant\left(\frac{v_{\varepsilon}\left|\nabla m_{\varepsilon}\right|^{2}}{1+v_{\varepsilon} m_{\varepsilon}}\right)^{\frac{1}{2}}\left|\nabla v_{\varepsilon}^{\frac{1}{2}}\right| \\
& \leqslant \frac{1}{2} \frac{v_{\varepsilon}\left|\nabla m_{\varepsilon}\right|^{2}}{1+v_{\varepsilon} m_{\varepsilon}}+\frac{1}{2}\left|\nabla v_{\varepsilon}^{\frac{1}{2}}\right|^{2} \\
& \left|\nabla m_{\varepsilon}\right| \frac{\left|\nabla v_{\varepsilon}^{\frac{1}{2}}\right|}{\left(1+v_{\varepsilon}^{\frac{1}{2}} m_{\varepsilon}\right)^{2}} \leqslant \frac{v_{\varepsilon}^{\frac{1}{2}}\left|\nabla m_{\varepsilon}\right|\left|\nabla v_{\varepsilon}^{\frac{1}{2}}\right|}{\left(1+v_{\varepsilon} m_{\varepsilon}\right)^{2}}+\frac{\left(1-v_{\varepsilon}\right)^{\frac{1}{2}}\left|\nabla m_{\varepsilon}\right|\left|\nabla v_{\varepsilon}^{\frac{1}{2}}\right|}{\left(1+v_{\varepsilon} m_{\varepsilon}\right)^{2}} \\
& \leqslant\left(\frac{v_{\varepsilon}\left|\nabla m_{\varepsilon}\right|^{2}}{1+v_{\varepsilon} m_{\varepsilon}}\right)^{\frac{1}{2}}\left|\nabla v_{\varepsilon}^{\frac{1}{2}}\right|+2\left|\nabla m_{\varepsilon}^{\frac{1}{2}}\right|\left(\left(1-v_{\varepsilon}\right) m_{\varepsilon}\left|\nabla v_{\varepsilon}^{\frac{1}{2}}\right|^{2}\right)^{\frac{1}{2}} \\
& \leqslant \frac{1}{2} \frac{v_{\varepsilon}\left|\nabla m_{\varepsilon}\right|^{2}}{1+v_{\varepsilon} m_{\varepsilon}}+\frac{1}{2}\left|\nabla v_{\varepsilon}^{\frac{1}{2}}\right|^{2}+\left|\nabla m_{\varepsilon}^{\frac{1}{2}}\right|^{2}+\left(1-v_{\varepsilon}\right) m_{\varepsilon}\left|\nabla v_{\varepsilon}^{\frac{1}{2}}\right|^{2}, \\
& \frac{v_{\varepsilon} c_{\varepsilon}\left|\nabla m_{\varepsilon}\right|}{1+v_{\varepsilon} c_{\varepsilon}} \frac{\left|\nabla v_{\varepsilon}^{\frac{1}{2}}\right|}{\left(1+v_{\varepsilon}^{\frac{1}{2}} m_{\varepsilon}\right)^{2}} \leqslant \frac{2 v_{\varepsilon}^{\frac{1}{2}} m_{\varepsilon}^{\frac{1}{2}}\left(m_{\varepsilon}+p_{\varepsilon}\right)^{\frac{1}{2}}}{\left(1+v_{\varepsilon}^{\frac{1}{2}} m_{\varepsilon}\right)\left(1+v_{\varepsilon} c_{\varepsilon}\right)^{\frac{1}{2}}}\left(\frac{v_{\varepsilon} c_{\varepsilon}\left|\nabla m_{\varepsilon}^{\frac{1}{2}}\right|^{2}}{1+v_{\varepsilon} c_{\varepsilon}}\right)^{\frac{1}{2}}\left|\nabla v_{\varepsilon}^{\frac{1}{2}}\right| \\
& \leqslant \frac{2 v_{\varepsilon}^{\frac{1}{2}} m_{\varepsilon}+2 C_{p}^{\frac{1}{2}}\left(v_{\varepsilon} m_{\varepsilon}\right)^{\frac{1}{2}}}{\left(1+v_{\varepsilon}^{\frac{1}{2}} m_{\varepsilon}\right)\left(1+v_{\varepsilon} m_{\varepsilon}\right)^{\frac{1}{2}}}\left(\frac{v_{\varepsilon} c_{\varepsilon}\left|\nabla m_{\varepsilon}^{\frac{1}{2}}\right|^{2}}{1+v_{\varepsilon} c_{\varepsilon}}\right)^{\frac{1}{2}}\left|\nabla v_{\varepsilon}^{\frac{1}{2}}\right| \\
& \leqslant C_{18} \frac{v_{\varepsilon} c_{\varepsilon}\left|\nabla m_{\varepsilon}^{\frac{1}{2}}\right|^{2}}{1+v_{\varepsilon} c_{\varepsilon}}+C_{18}\left|\nabla v_{\varepsilon}^{\frac{1}{2}}\right|^{2}
\end{aligned}
$$




$$
v_{\varepsilon}^{\frac{1}{2}} m_{\varepsilon}\left|\nabla v_{\varepsilon}^{\frac{1}{2}}\right| \frac{\left|\nabla v_{\varepsilon}^{\frac{1}{2}}\right|}{\left(1+v_{\varepsilon}^{\frac{1}{2}} m_{\varepsilon}\right)^{2}} \leqslant\left|\nabla v_{\varepsilon}^{\frac{1}{2}}\right|^{2} .
$$

Combining (66)-(72) with (24)-(26), (28), (29), (31), we obtain that

$$
\left\|q_{\varepsilon} \cdot \nabla \frac{v_{\varepsilon}^{\frac{1}{2}}}{1+v_{\varepsilon}^{\frac{1}{2}} m_{\varepsilon}}\right\|_{L^{1}\left(0, T ; L^{1}(\Omega)\right)} \leqslant C_{19}(T) .
$$

Therefore, (62) together with (63), (65) and (73) yield that

$$
\left\|\frac{v_{\varepsilon}^{\frac{1}{2}} \partial_{t} m_{\varepsilon}}{1+v_{\varepsilon}^{\frac{1}{2}} m_{\varepsilon}}\right\|_{L^{1}\left(0, T ; W^{-1,1}(\Omega)\right)} \leqslant C_{20}(T) .
$$

Finally, with the help of estimates (61) and (74), we obtain from (59) that

$$
\left\|\partial_{t} u_{\varepsilon}\right\|_{L^{1}\left(0, T ; W^{-1,1}(\Omega)\right)} \leqslant C_{21}(T) .
$$

Estimates for $m_{\varepsilon}$ in $(0, T) \times \operatorname{int}\left\{v_{\varepsilon_{3} 0}=0\right\}$

While studying the function $m_{\varepsilon}$, the auxiliary function $u_{\varepsilon}$ introduced in (51) is of use only in the set $\left\{v_{\varepsilon}>0\right\}$. It clearly reveals no further information about the behaviour of $m_{\varepsilon}$ over the level sets $\left\{v_{\varepsilon}(t)=0\right\}, t \in(0, T)$, each of whom almost coincide with $\left\{v_{\varepsilon_{3} 0}=0\right\}$. The latter is to mean that $\left\{v_{\varepsilon}(t)=0\right\}$ differs from $\left\{v_{\varepsilon_{3} 0}=0\right\}$ by a null set and is thus preserved in time. In order to see this, let us divide both sides of the $\operatorname{ODE}(3 \mathrm{c})$ by $v_{\varepsilon}$ and integrate over $(0, t)$ for arbitrary $t \in(0, T)$. We obtain that

$$
\ln \left(v_{\varepsilon}(t)\right)-\ln \left(v_{\varepsilon_{3} 0}\right)=\int_{0}^{t} \mu_{v}\left(1-v_{\varepsilon}\right) d t-\lambda \int_{0}^{t} m_{\varepsilon} d t .
$$

Since $0 \leqslant v_{\varepsilon} \leqslant 1$ and $m_{\varepsilon} \in L^{1}\left(0, T ; L^{1}(\Omega)\right)$, the right-hand side of (76) is finite a.e. in $\Omega$. Hence, the same holds for the left-hand side of (76). But this means that for all $t \in \mathbb{R}^{+}$it necessarily holds that

$$
\begin{aligned}
& v_{\varepsilon}(t)>0 \text { a.e. in }\left\{v_{\varepsilon_{3} 0}>0\right\}, \\
& v_{\varepsilon}(t)=0 \text { a.e. in }\left\{v_{\varepsilon_{3} 0}=0\right\} .
\end{aligned}
$$

Similarly, we obtain from the original equation (1c) that

$$
\begin{aligned}
& v(t)>0 \text { a.e. in }\left\{v_{0}>0\right\}, \\
& v(t)=0 \text { a.e. in }\left\{v_{0}=0\right\} .
\end{aligned}
$$

Observe that, at least in $(0, T) \times \operatorname{int}\left\{v_{\varepsilon_{3} 0}=0\right\}, m_{\varepsilon}$ solves the linear initial value problem

$$
\begin{array}{ll}
\partial_{t} m_{\varepsilon}=\varepsilon_{1} \Delta m_{\varepsilon}-\alpha m_{\varepsilon} & \text { in } \mathbb{R}^{+} \times \operatorname{int}\left\{v_{\varepsilon_{3} 0}=0\right\}, \\
m_{\varepsilon}(0)=m_{\varepsilon_{2} 0} & \text { in } \operatorname{int}\left\{v_{\varepsilon_{3} 0}=0\right\} .
\end{array}
$$

Combining (31) and (39), we conclude from (79a) that

$$
\left\|\partial_{t} m_{\varepsilon}\right\|_{L^{1}\left(0, T ; W^{-1,1}\left(\operatorname{int}\left\{v_{\varepsilon_{3} 0}=0\right\}\right)\right)} \leqslant C_{22}(T) .
$$

Since $m_{\varepsilon_{2} 0}$ is smooth, $m_{\varepsilon}$ is a classical solution to (79a). Differentiating (79a) with respect to $x_{i}, i \in\{1, \ldots, N\}$, we obtain that

$$
\partial_{t} \partial_{x_{i}} m_{\varepsilon}=\varepsilon_{1} \Delta \partial_{x_{i}} m_{\varepsilon}-\alpha \partial_{x_{i}} m_{\varepsilon}
$$

Let now $\varphi$ be some smooth cut-off function with $\operatorname{supp} \varphi \subset \operatorname{int}\left\{v_{\varepsilon_{3} 0}=0\right\}$ and let $a \in(1,2)$, the latter to be specified below. Multiplying (81) by $a \varphi^{2}\left|\partial_{x_{i}} m_{\varepsilon}\right|^{a-2} \partial_{x_{i}} m_{\varepsilon}$ and integrating by parts over $\Omega$, we obtain with the Hölder and Young inequalities that

$$
\begin{aligned}
\frac{d}{d t}\left\|\varphi\left|\partial_{x_{i}} m_{\varepsilon}\right|^{\frac{a}{2}}\right\|^{2} & =-\frac{4(a-1)}{a} \varepsilon_{1}\left\|\varphi \nabla\left|\partial_{x_{i}} m_{\varepsilon}\right|^{\frac{a}{2}}\right\|^{2}-4 \varepsilon_{1}\left(\varphi \nabla\left|\partial_{x_{i}} m_{\varepsilon}\right|^{\frac{a}{2}},\left|\partial_{x_{i}} m_{\varepsilon}\right|^{\frac{a}{2}} \nabla \varphi\right)-a \alpha\left\|\varphi\left|\partial_{x_{i}} m_{\varepsilon}\right|^{\frac{a}{2}}\right\|^{2} \\
& \leqslant C_{23}(a)\|\nabla \varphi\|_{L^{\infty}(\Omega)}^{2} \varepsilon_{1}\left\|\partial_{x_{i}} m_{\varepsilon}\right\|_{a}^{a} \\
& =C_{24}(a)\|\nabla \varphi\|_{L^{\infty}(\Omega)}^{2} \varepsilon_{1}\left\|m_{\varepsilon}^{\frac{1}{2}}\left|\partial_{x_{i}} m_{\varepsilon}^{\frac{1}{2}}\right|\right\|_{a}^{a} \\
& \leqslant C_{25}(a)\|\nabla \varphi\|_{L^{\infty}(\Omega)}^{2} \varepsilon_{1}\left\|m_{\varepsilon}^{\frac{1}{2}}\right\|_{\frac{1}{\frac{1}{a}-\frac{1}{2}}}^{a}\left\|\partial_{x_{i}} m_{\varepsilon}^{\frac{1}{2}}\right\|^{a} .
\end{aligned}
$$


Owing to a Sobolev interpolation inequality it holds that

$$
\left\|m_{\varepsilon}^{\frac{1}{2}}\right\|_{\frac{1}{a}-\frac{1}{2}} \leqslant C_{26}(a)\left(\left\|\nabla m_{\varepsilon}^{\frac{1}{2}}\right\|+\left\|m_{\varepsilon}^{\frac{1}{2}}\right\|\right)^{N\left(1-\frac{1}{a}\right)}\left\|m_{\varepsilon}^{\frac{1}{2}}\right\|^{1-N\left(1-\frac{1}{a}\right)} \text { for } a \in\left[1, \frac{N}{N-1}\right] .
$$

Integrating (82) over $(0, t)$ and using (28), (31), (83) and the Hölder inequality, we thus obtain that

$$
\left\|\varphi\left|\nabla m_{\varepsilon}(t)\right|^{\frac{a}{2}}\right\|^{2} \leqslant\left\|\varphi\left|\nabla m_{\varepsilon_{2} 0}\right|^{\frac{a}{2}}\right\|^{2}+C_{27}(a, T)\|\nabla \varphi\|_{L^{\infty}(\Omega)}^{2} \varepsilon_{1}^{1-\frac{N}{2}(a-1)-\frac{a}{2}} \text { for } a \in\left(1, \frac{N}{N-1}\right] .
$$

The first term on right-hand side of (84) doesn't depend upon $\varepsilon_{1}$, while the second one converges to zero for $a \in\left(1, \frac{N+2}{N+1}\right) \subset$ $\left(1, \frac{N}{N-1}\right]$. Therefore, we obtain from (84) in the limit as $\varepsilon_{1} \rightarrow 0$ that

$$
\begin{aligned}
\limsup _{\varepsilon_{1} \rightarrow 0}\left\|\varphi\left|\nabla m_{\varepsilon}(t)\right|^{\frac{a}{2}}\right\|^{2} & \leqslant\left\|\varphi\left|\nabla m_{\varepsilon_{2} 0}\right|^{\frac{a}{2}}\right\|^{2} \\
& \leqslant\left\|\nabla m_{\varepsilon_{2} 0}\right\|_{L^{\infty}(\Omega)}^{a} \text { for } a \in\left(1, \frac{N+2}{N+1}\right) .
\end{aligned}
$$

Since $\varphi$ was an arbitrary cut-off function with $\operatorname{supp} \varphi \subset \operatorname{int}\left\{v_{\varepsilon_{3} 0}=0\right\}$, (85) yields that

$$
\limsup _{\varepsilon_{1} \rightarrow 0}\left\|\nabla m_{\varepsilon}(t)\right\|_{L^{a}\left(\operatorname{int}\left\{v_{\varepsilon_{3} 0}=0\right\}\right)} \leqslant\left\|\nabla m_{\varepsilon_{2} 0}\right\|_{L^{\infty}(\Omega)} \text { for } a \in\left(1, \frac{N+2}{N+1}\right) .
$$

Together with (31), (86) yields that

$$
\limsup _{\varepsilon_{1} \rightarrow 0}\left\|m_{\varepsilon}(t)\right\|_{W^{1,1}\left(\operatorname{int}\left\{v_{\varepsilon_{3} 0}=0\right\}\right)} \leqslant C_{28}\left(\varepsilon_{2}\right)
$$

\section{Global existence for the original problem}

In this section we aim to pass to the limit in (3) in order to obtain a solution of the original problem.

Remark 7.1 (Vector notation) Let $\left\{\varepsilon_{i, n_{i}}\right\} \subset(0,1), i=1,2,3$, be three sequences. In this section, we make use of the following vector notation:

$$
n_{i: 3}:=\left(n_{i}, \ldots, n_{3}\right), \varepsilon_{n_{i: 3}}:=\left(\varepsilon_{i, n_{i}}, \ldots, \varepsilon_{3, n_{3}}\right), i=1,2 .
$$

Owing to the estimates obtained in the preceding section, we are now in a position where we can establish a list convergences (see below) holding jointly for some sequences

$$
\varepsilon_{i, n_{i}} \underset{n_{i} \rightarrow \infty}{\rightarrow} 0, i=1,2,3
$$

Convergence for the initial data

Due to (12)-(14) it holds that

$$
\begin{aligned}
& m_{\varepsilon_{2, n_{2}} 0} \underset{n_{2} \rightarrow \infty}{\rightarrow} m_{0} \text { in } L^{1}(\Omega) \text { and a.e. in } \Omega, \\
& p_{\varepsilon_{2, n_{2} 0} 0}^{\underset{n_{2} \rightarrow \infty}{\rightarrow}} p_{0} \text { in } L^{\infty}(\Omega) \text { and a.e. in } \Omega, \\
& v_{\varepsilon_{3, n_{3}} 0}^{\frac{1}{2}} \underset{n_{3} \rightarrow \infty}{\rightarrow} v_{0}^{\frac{1}{2}} \text { in } L^{2}(\Omega) \text { and a.e. in } \Omega
\end{aligned}
$$

Convergence for $\left\{v_{\varepsilon_{n_{1: 3}}}\right\}$

It holds that: due to (24), (50) and a version of the Lions-Aubin Lemma [35, Corollary 4]

$$
v_{\varepsilon_{n 1: 3}}^{\frac{1}{2}} \underset{n_{1: 3} \rightarrow \infty}{\rightarrow} v^{\frac{1}{2}} \text { in } L^{2}\left(0, T ; L^{2}(\Omega)\right) ;
$$

due to $(91)$

$$
v_{\varepsilon_{n_{1: 3}}}^{\frac{1}{2}} \underset{n_{1: 3} \rightarrow \infty}{\rightarrow} v^{\frac{1}{2}} \text { a.e. in }(0, T) \times \Omega
$$


due to (92)

$$
v_{\varepsilon_{n_{1: 3}}} \underset{n_{1: 3} \rightarrow \infty}{\rightarrow} v \text { a.e. in }(0, T) \times \Omega
$$

due to (93) and the dominated convergence theorem

$$
v_{\varepsilon_{n_{1: 3}}}^{a} \underset{n_{1: 3} \rightarrow \infty}{\rightarrow} v^{a} \text { in } L^{p}((0, T) \times \Omega) \text { and a.e. in }(0, T) \times \Omega \text { for all } a>0, p \geqslant 1
$$

due to (24), (91) and the Banach-Alaoglu theorem

$$
\nabla v_{\varepsilon_{n 1: 3}}^{\frac{1}{2}} \underset{n_{1: 3} \rightarrow \infty}{\longrightarrow} \nabla v^{\frac{1}{2}} \text { in } L^{2}\left(0, T ; L^{2}(\Omega)\right)
$$

Convergence for $\left\{m_{\varepsilon_{n_{1: 3}}}\right\}$ in $(0, T) \times\left\{v_{0}>0\right\}$

It holds that: due to (58), (75) and a version of the Lions-Aubin Lemma [35, Corollary 4]

$$
\ln \left(1+v_{\varepsilon_{n_{1: 3}}}^{\frac{1}{2}} m_{\varepsilon_{n_{1: 3}}}\right) \underset{n_{1: 3} \rightarrow \infty}{\rightarrow} u \text { in } L^{1}\left(0, T ; L^{1}(\Omega)\right)
$$

due to (96)

$$
\ln \left(1+v_{\varepsilon_{n_{1: 3}}}^{\frac{1}{2}} m_{\varepsilon_{n_{1: 3}}}\right) \underset{n_{1: 3} \rightarrow \infty}{\rightarrow} u \text { a.e. in }(0, T) \times \Omega
$$

due to (97)

$$
v_{\varepsilon_{n_{1: 3}}}^{\frac{1}{2}} m_{\varepsilon_{n_{1: 3}}} \underset{n_{1: 3} \rightarrow \infty}{\rightarrow} e^{u}-1=: w \text { a.e. in }(0, T) \times \Omega
$$

due to (93), (98)

$$
m_{\varepsilon_{n_{1: 3}}} \underset{n_{1: 3} \rightarrow \infty}{\rightarrow} \frac{w}{v^{\frac{1}{2}}}=: m \text { a.e. in }(0, T) \times\left\{v_{0}>0\right\}
$$

due to (32), (99) and the Vitali convergence theorem

$$
m_{\varepsilon_{n_{1: 3}}} \underset{n_{1: 3} \rightarrow \infty}{\rightarrow} m \text { in } L^{1}\left((0, T) \times\left\{v_{0}>0\right\}\right) .
$$

Convergence for $\left\{m_{\varepsilon_{n_{1: 3}}}\right\}$ in $(0, T) \times\left\{v_{0}=0\right\}$

It holds due to (80), (87) and a version of the Lions-Aubin Lemma [35, Corollary 4] that

$$
m_{\varepsilon_{n_{1: 3}}} \underset{n_{1} \rightarrow \infty}{\rightarrow} m_{n_{2: 3}} \text { in } L^{1}\left((0, T) \times \operatorname{int}\left\{v_{\varepsilon_{3, n_{3}} 0}=0\right\}\right),
$$

and so we may pass to the distributional limit in (79):

$$
\begin{aligned}
& \partial_{t} m_{n_{2: 3}}=-\alpha m_{n_{2: 3}} \text { in } \mathbb{R}^{+} \times \operatorname{int}\left\{v_{\varepsilon_{3, n_{3}} 0}=0\right\}, \\
& m_{n_{2: 3}}(0)=m_{\varepsilon_{2, n_{2}} 0} \text { in } \operatorname{int}\left\{v_{\varepsilon_{3, n_{3}} 0}=0\right\} .
\end{aligned}
$$

Due to (88) and the continuous dependence of solutions of an ODE with smooth coefficients upon the initial data, it follows with (102b) that

$$
m_{n_{2: 3}} \underset{n_{2} \rightarrow \infty}{\rightarrow} m_{n_{3}} \text { in } L^{1}\left((0, T) \times \operatorname{int}\left\{v_{\varepsilon_{3, n_{3} 0}}=0\right\}\right)
$$

and

$$
m_{n_{3}}=m \text { a.e. in }(0, T) \times \operatorname{int}\left\{v_{\varepsilon_{3, n_{3}} 0}=0\right\} \cap\left\{v_{0}=0\right\}
$$

where $m$ solves

$$
\begin{aligned}
& \partial_{t} m=-\alpha m \text { in } \mathbb{R}^{+} \times\left\{v_{0}=0\right\}, \\
& m(0)=m_{0} \text { in }\left\{v_{0}=0\right\} .
\end{aligned}
$$


Combining (101), (103)-(105), we conclude that

$$
m_{\varepsilon_{n_{1: 3}}} \underset{n_{1} \rightarrow \infty n_{2} \rightarrow \infty}{\rightarrow} m \text { in } L^{1}\left((0, T) \times \operatorname{int}\left\{v_{\varepsilon_{3, n_{3}} 0}=0\right\} \cap\left\{v_{0}=0\right\}\right),
$$

hence also

$$
m_{\varepsilon_{n_{1: 3}}} \underset{n_{1} \rightarrow \infty n_{2} \rightarrow \infty}{\rightarrow} m \text { on }(0, T) \times \operatorname{int}\left\{v_{\varepsilon_{3, n_{3}} 0}=0\right\} \cap\left\{v_{0}=0\right\} \text { in the measure }
$$

Together with property (15), (107) yields that

$$
\lim _{n_{3} \rightarrow \infty} \limsup _{n_{2} \rightarrow \infty} \limsup _{n_{1} \rightarrow \infty}\left|\left\{\left|m_{\varepsilon_{n_{1: 3}}}-m\right| \geqslant \delta\right\}\right|=0 \text { on }(0, T) \times\left\{v_{0}=0\right\} \text { for all } \delta>0 .
$$

Finally, combining (32), (108) and using the Vitali convergence theorem, we arrive at

$$
\lim _{n_{3} \rightarrow \infty} \limsup _{n_{2} \rightarrow \infty} \limsup _{n_{1} \rightarrow \infty}\left\|m_{\varepsilon_{n_{1: 3}}}-m\right\|_{L^{1}\left((0, T) \times\left\{v_{0}=0\right\}\right)}=0 .
$$

Convergence for $p_{\varepsilon_{n_{1: 3}}}$ in (3b)-(3c)

We may consider (3b)-(3c) together with the corresponding initial conditions as an abstract ODE system with respect to the variables $p_{\varepsilon_{n 1: 3}}$ and $v_{\varepsilon_{n_{1: 3}}}$ regarding $m_{\varepsilon_{n_{1: 3}}}$ as a parameter function:

$$
\frac{d}{d t}\left(\begin{array}{c}
p_{\varepsilon_{n_{1: 3}}} \\
v_{\varepsilon_{n_{1: 3}}}
\end{array}\right)=G\left(\left(\begin{array}{c}
p_{\varepsilon_{n_{1: 3}}} \\
v_{\varepsilon_{n_{1: 3}}}
\end{array}\right), m_{\varepsilon_{n_{1: 3}}}\right) \text { in } L^{1}(\Omega),
$$

where the function $G:\left(\left[0, C_{p}\right] \times[0,1]\right) \times \mathbb{R}_{0}^{+} \rightarrow \mathbb{R}_{0}^{+}$is clearly globally Lipschitz. Here $C_{p}$ is an upper bound for the family $\left\{p_{\varepsilon}\right\}$, compare (21). Using the standard abstract ODE theory in $L^{1}$, which states that the solutions depend continuously upon parameters and initial data, we conclude with (89)-(90) and (100), (109) that

$$
\begin{aligned}
& p_{\varepsilon_{n_{1: 3}}} \underset{n_{1: 3} \rightarrow \infty}{\rightarrow} p \text { in } L^{1}\left((0, T) \times\left\{v_{0}>0\right\}\right), \\
& p_{\varepsilon_{n_{1: 3}} \underset{n_{1: 3} \rightarrow \infty}{\rightarrow}}^{\rightarrow} p \text { a.e. in }(0, T) \times\left\{v_{0}>0\right\}, \\
& \lim _{n_{3} \rightarrow \infty} \limsup _{n_{2} \rightarrow \infty} \limsup _{n_{1} \rightarrow \infty}\left\|p_{\varepsilon_{n_{1: 3}}}-p\right\|_{L^{1}\left((0, T) \times\left\{v_{0}=0\right\}\right)}=0,
\end{aligned}
$$

and $m, p, v$ solve the original equations (1b)-(1c) and satisfy the initial conditions in $L^{1}$-sense, as stated in Definition 4.2 .

\section{Convergence in (4)}

In order to finish the proof of Theorem 4.5, it remains to check that the triple $(m, p, v)$, which we obtained above by means of our limit procedure, satisfies the weak formulation (2). For this purpose, we need to pass to the limit in the weak formulation (4). Taking $\varepsilon:=\varepsilon_{n_{1: 3}}$, we have that

$$
\begin{aligned}
& \int_{\Omega} m_{\varepsilon_{2, n_{2}} 0} \varphi d x \psi(0)-\int_{0}^{T} \int_{\Omega} m_{\varepsilon_{n_{1: 3}}} \varphi d x \psi^{\prime} d t \\
& =\int_{0}^{T} \int_{\Omega}-\varepsilon_{n_{1: 3}} \nabla m_{\varepsilon_{n_{1: 3}}} \cdot \nabla \varphi \psi-I_{\varepsilon_{n_{1: 3}}} \cdot \nabla \varphi \psi+\kappa_{v} \nabla\left(\int_{0}^{t} \frac{v_{\varepsilon_{n_{1: 3}}} m_{\varepsilon_{n_{1: 3}}}}{1+v_{\varepsilon_{n_{1: 3}}}} d \tau\right) \cdot \nabla \varphi \psi^{\prime} \\
& \quad+\left(-\alpha m_{\varepsilon_{n_{1: 3}}}+\beta v_{\varepsilon_{n_{1: 3}}} p_{\varepsilon_{n_{1: 3}}}\right) \varphi \psi d x d t,
\end{aligned}
$$

where in order to shorten the notation we introduced

$$
\begin{aligned}
I_{\varepsilon_{n_{1: 3}}}:= & \left(\frac{\kappa_{m} c_{\varepsilon_{n_{1: 3}}}}{1+v_{\varepsilon_{n_{1: 3}}} c_{\varepsilon_{n_{1: 3}}}}+\frac{\kappa_{v}}{1+v_{\varepsilon_{n_{1: 3}}}}\right) 2 v_{\varepsilon_{n_{1: 3}}}^{\frac{1}{2}}\left(m_{\varepsilon_{n_{1: 3}}}+1\right)^{\frac{1}{2}} \\
& \cdot\left(\nabla\left(v_{\varepsilon_{n_{1: 3}}}^{\frac{1}{2}}\left(m_{\varepsilon_{n_{1: 3}}}+1\right)^{\frac{1}{2}}\right)-\left(m_{\varepsilon_{n_{1: 3}}}+1\right)^{\frac{1}{2}} \nabla v_{\varepsilon_{n_{1: 3}}}^{\frac{1}{2}}\right) \\
= & \frac{\kappa_{m} v_{\varepsilon_{n_{1: 3}} c_{\varepsilon_{n_{1: 3}}}}}{1+v_{\varepsilon_{n_{1: 3}}} c_{\varepsilon_{n_{1: 3}}}} \nabla m_{\varepsilon_{n_{1: 3}}}+\frac{\kappa_{v} v_{\varepsilon_{n_{1: 3}}}}{1+v_{\varepsilon_{n_{1: 3}}}} \nabla m_{\varepsilon_{n_{1: 3}}} .
\end{aligned}
$$

Observe that the representations (114) and (115) coincide due to the chain and product rules. But for $I_{\varepsilon_{n_{1: 3}}}$, the convergence of the terms in (113) can be obtained with standard tools using (21), (32), (39), (88), (92), (93), (110). We thus leave these details aside and concentrate on the weak $L^{1}$-limit for $I_{\varepsilon_{n_{1: 3}}}$. To start with, (41), (45) and (115) imply that

$$
\left\{I_{\varepsilon_{n_{1: 3}}}\right\} \text { is uniformly integrable in }(0, T) \times \Omega \text {. }
$$


Hence, the Dunford-Pettis theorem applies and yields the existence of such limit:

$$
I_{\varepsilon_{n_{1: 3}}} \underset{n_{1: 3} \rightarrow \infty}{\longrightarrow} \tilde{I} \text { in } L^{1}((0, T) \times \Omega) .
$$

We claim that $\tilde{I}$ can be obtained by simply dropping the index $\varepsilon_{n_{1: 3}}$ everywhere in (114). We observe that (114) admits the following reformulation:

$$
I_{\varepsilon_{n_{1: 3}}}=I_{1}\left(m_{\varepsilon_{n_{1: 3}}}, p_{\varepsilon_{n_{1: 3}}}, v_{\varepsilon_{n_{1: 3}}}\right) \nabla\left(I_{3}\left(m_{\varepsilon_{n_{1: 3}}}, v_{\varepsilon_{n_{1: 3}}}\right)\right)+I_{2}\left(m_{\varepsilon_{n_{1}: 3}}, p_{\varepsilon_{n_{1: 3}}}, v_{\varepsilon_{n_{1}: 3}}\right) \nabla v_{\varepsilon_{n_{1: 3}}}^{\frac{1}{2}},
$$

where $I_{1}, I_{2}: \mathbb{R}^{3} \mapsto \mathbb{R}, I_{3}: \mathbb{R}^{2} \mapsto \mathbb{R}$ are continuous functions. Since $I_{1}(\cdot, \cdot, 0) \equiv I_{2}(\cdot, \cdot, 0) \equiv 0$, it holds with (77), (118) that

$$
I_{\varepsilon_{n_{1: 3}}}=0 \text { a.e. in }(0, T) \times\left\{v_{\varepsilon_{3} 0}=0\right\} .
$$

Combining (116), (119) with (77), (78) and property (15) and passing to the limit in the measure on $(0, T) \times\left\{v_{0}=0\right\}$, we obtain that, as expected,

$$
\tilde{I}=0 \text { a.e. in }(0, T) \times\left\{v_{0}=0\right\} .
$$

Further, we have due to (92), (99), (111), and the continuity of $I_{1}, I_{2}, I_{3}$ that

$$
\begin{aligned}
& I_{1}\left(m_{\varepsilon_{n_{1: 3}}}, p_{\varepsilon_{n_{1: 3}}}, v_{\varepsilon_{n_{1: 3}}}\right) \underset{n_{1: 3 \rightarrow \infty} \rightarrow}{\rightarrow} I_{1}(m, p, v) \text { a.e. in }(0, T) \times\left\{v_{0}>0\right\}, \\
& I_{2}\left(m_{\varepsilon_{n_{1: 3}}}, p_{\varepsilon_{n_{1: 3}}}, v_{\varepsilon_{n_{1: 3}}}\right) \underset{n_{1: 3} \rightarrow \infty}{\rightarrow} I_{2}(m, p, v) \text { a.e. in }(0, T) \times\left\{v_{0}>0\right\}, \\
& I_{3}\left(m_{\varepsilon_{n_{1: 3}}}, v_{\varepsilon_{n_{1: 3}}}\right) \underset{n_{1: 3} \rightarrow \infty}{\rightarrow} I_{3}(m, v) \text { a.e. in }(0, T) \times\left\{v_{0}>0\right\} .
\end{aligned}
$$

Using (33), (122) and the Vitali convergence theorem, we obtain that

$$
I_{3}\left(m_{\varepsilon_{n_{1: 3}}}, v_{\varepsilon_{n_{1: 3}}}\right) \underset{n_{1: 3} \rightarrow \infty}{\rightarrow} I_{3}(m, v) \text { in } L^{1}\left((0, T) \times\left\{v_{0}>0\right\}\right) .
$$

Together with (35), this yields by using the Dunford-Pettis theorem that

$$
\nabla\left(I_{3}\left(m_{\varepsilon_{n_{1: 3}}}, v_{\varepsilon_{n_{1: 3}}}\right)\right) \underset{n_{1: 3} \rightarrow \infty}{\rightarrow} \nabla\left(I_{3}(m, v)\right) \text { in } L^{1}\left((0, T) \times\left\{v_{0}>0\right\}\right) .
$$

Finally, combining (95), (120), (121), (124) and using Lemma A.3, we arrive at

$$
\begin{aligned}
& I_{1}\left(m_{\varepsilon_{n_{1}: 3}}, p_{\varepsilon_{n_{1: 3}}}, v_{\varepsilon_{n_{1}: 3}}\right) \nabla\left(I_{3}\left(m_{\varepsilon_{n_{1}: 3}}, v_{\varepsilon_{n_{1}: 3}}\right)\right)+I_{2}\left(m_{\varepsilon_{n_{1}: 3}}, p_{\varepsilon_{n_{1: 3}}}, v_{\varepsilon_{n_{1: 3}}}\right) \nabla v_{\varepsilon_{n_{1}: 3}}^{\frac{1}{2}} \\
& \underset{n_{1: 3} \rightarrow \infty}{\longrightarrow} I_{1}(m, p, v) \nabla\left(I_{3}(m, v)\right)+I_{2}(m, p, v) \nabla v^{\frac{1}{2}} \text { in } L^{1}\left((0, T) \times\left\{v_{0}>0\right\}\right) .
\end{aligned}
$$

The proof of Theorem 4.5 is thus completed.

\section{Numerical Simulations}

We discretize the PDE-ODE-ODE system (1) using a local mass conservative and monotone finite volume method. We use the software package Dune $[36,37,38,39,40]$ with Dune-PDELab and consider on the domain $\Omega=(0,1)^{2}$ the structured quadrilateral grid Yaspgrid therein.

\subsection{Implementation}

Let $\mathcal{C}$ be the set of computational cells in the grid and denote by $\mathcal{E}(c)$ the (inner) edges of the grid surrounding a cell $c$. Then we approximate the vector $u=(m, p, v)^{T}$ in the space $\mathcal{P}_{0}^{3}$, so the restriction of $u$ on a computational cell $c$ is a constant vector. The discretization in space now takes place with the aid of two-point flux approximations as in [41]. First we define the diffusion coefficient $D(u)=\frac{\kappa_{m} u_{3}\left(u_{1}+u_{2}\right)}{1+u_{3}\left(u_{1}+u_{2}\right)}$ and the drift velocity $V(u)=\frac{\kappa_{v}}{\left(1+u_{1}+u_{2}\right)^{2}}$. The convection velocity $V(u) \nabla u_{3}$ and the diffusion term $D(u) \nabla u_{1}$ have both the same structure, therefore we use the same space discretization. Hence we will only present the diffusive flux discretization in detail. We may integrate

$$
\partial_{t} u_{1}=\nabla \cdot\left(D(u) \nabla u_{1}-V(u) \nabla u_{3} u_{1}\right)
$$

over a computational cell $c$ by employing the Gauß theorem

$$
\left.\partial_{t}\left(u_{1}\right)\right|_{c}=\sum_{e \in \mathcal{E}(c)} F_{c}^{e}+V_{c}^{e}\left(u_{1}\right)_{e}^{+},
$$


where $F^{e}$ is the approximation of the diffusive flux and $V^{e}$ is an approximation of the drift velocity though an edge $e$. The symbol $\left(u_{1}\right)_{e}^{+}$stands for a simple upwinding scheme [41]. To get a locally mass conservative method, we require that for each edge $e$ between cells $c$ and $c^{\prime}$ we have $F_{c}^{e}+F_{c^{\prime}}^{e}=0$, as well as $V_{c}^{e}+V_{c^{\prime}}^{e}=0$. This gives the possibility to resolve the edge variables and for an edge $e$ between $c$ and $c^{\prime}$ we have

$$
F_{c}^{e}=\frac{\left.\left.D(u)\right|_{c} D(u)\right|_{c^{\prime}}}{\left.D(u)\right|_{c}+\left.D(u)\right|_{c^{\prime}}}\left(\left.\left(u_{1}\right)\right|_{c^{\prime}}-\left.\left(u_{1}\right)\right|_{c}\right) \frac{2|e|}{d\left(c, c^{\prime}\right)} .
$$

The drift velocity is computed in the same way. Due to the diffusion, we need to employ an implicit method in order to circumvent a hard restriction on the time step. So the discretized scheme reads

$$
u^{k+1}+\Delta t \Phi\left(u^{k+1}\right)=u^{k}
$$

where $\Phi$ represents the diffusive and convective flux as well as the reaction term. We solve the previous equation (125) by the classical Newton method, where the necessary differentiation of the nonlinear flux $\Phi$ is done numerically.

Remark 8.1 (Convergence) We would like to point out that our numerical simulations were performed by a usual finite volume method, whereas the general convergence theory for this kind of numerical schemata is well-developed only for nondegenerate parabolic problems. It goes far beyond the aims of this paper to investigate the convergence in our case, although it might be an interesting issue. By no means, however, is this a trivial task. Our simulations are only meant to be an illustration of how -according to this new model- the solution behaviour could possibly look like.

\subsection{Results}

We have to select initial conditions. Therefore we assume a grate-like initial condition for $v$ and define the following sets:

$$
\begin{aligned}
& S_{1}=\left\{x \in \mathbb{R}^{2} \mid x_{2} \in(0.35,0.45)\right\} \\
& S_{2}=\left\{x \in \mathbb{R}^{2} \mid x_{2} \in(0.7,0.8)\right\} \\
& S_{3}=\left\{x \in \mathbb{R}^{2}|| x_{1}-\hat{x} \mid<0.015, \text { for } \hat{x} \in\{0.4,0.45,0.5,0.55,0.6,0.65\}\right\} \\
& S_{4}=\left\{x \in \mathbb{R}^{2}|| x_{1}-x_{2}-\hat{x} \mid<0.01, \text { for } \hat{x} \in\{-0.2,-0.1,0.0\}\right\} \\
& S_{5}=\left\{x \in \mathbb{R}^{2}|| x_{1}-0.5 \cdot x_{2}-\hat{x} \mid<0.01, \text { for } \hat{x} \in\{0.5,0.6\}\right\}
\end{aligned}
$$

In $[26,21]$ we used a uniform distribution to characterize the initial ECM density. Here, however, we want to see the effects of a fibrous tissue on the tumor invasion and therefore we select the initial configuration of ECM fibers in a rather intuitive way as

$$
\left.v_{0}(x)=0.95 \cdot 1{ }_{\left\{x \in \bigcup_{i=1}^{5} S_{i}\right.}\right\}^{+0.05}
$$

resembling high density fiber tracts with a surrounding tissue of lower density. Now we need to think about the initial conditions for the tumor variables. We observe that migrating tumor cells (variable $m$ ) will pass into the proliferating regime if no tissue is available (at least it is highly improbable to find migrating cells in absence of tissue fibers) and will start to move otherwise. We assume that at the initial time there are no moving, but only resting and hence proliferating cells. For the initial population of proliferating tumor cells we selected an unsymmetric tumor bulk centered on fiber tracts as seen in Figure 1 . With all these considerations we select the initial conditions for the cell variables in the form

$$
\begin{aligned}
& m_{0}(x)=0.0 \\
& p_{0}(x)=\min \left\{\Psi _ { 0 . 0 2 } \left(x-(0.5,0.5)^{T}+0.5 \cdot \Psi_{0.01}\left(x-(0.45,0.49)^{T}\right)+0.5 \cdot \Psi_{0.01}\left(x-(0.52,0.5)^{T}, 5.0\right\},\right.\right.
\end{aligned}
$$

where

$$
\Psi_{\sigma}(s)=\frac{1}{2 \pi \sigma} \exp \left(-\frac{s^{2}}{2 \sigma^{2}}\right)
$$

The remaining task is to choose the parameters involved in the model. We select -in order to enable a comparison of the model (1) to the model in [26]- as many parameters as possible to be equal to the ones in the previous papers [17, 26, 21]. The tissue is distributed in a quite inhomogeneous way, however on a tissue fiber (or fiber bundle) the material is homogeneous, meaning that the tissue gradient $\nabla v$ and whence the haptotaxis is vanishing. Nevertheless, haptotaxis is not negligible, as it describes the guidance of cell migration by the tissue fibers (dissolved or not). The concrete parameter selection is summarized in Table 1 .

The grid we use is a triangulation of the unit cube in two dimensions, with 200 cells in each direction. So we also have to select a small time step $\Delta t$. In our calculations we used $\Delta t=0.5$ and performed simulations up to $t=50$.

Figure 2 shows the simulation results. The comparison between the evolution of migrating and proliferating cells elicits the expected behavior: the migrating cells are predominant in the regions with high tissue density (it can be actually seen how they follow the 


\begin{tabular}{|c|c|c||c|c|c|}
\hline Parameter & Value & Source & Parameter & Value & Source \\
\hline$\alpha$ & 0.05 & estimated & $\mu_{p}$ & 0.3 & {$[26,21]$} \\
$\beta$ & 0.1 & estimated & $\mu_{v}$ & 0.02 & {$[26,21]$} \\
$\kappa_{m}$ & $10^{-3}$ & {$[26,21]$} & $\eta$ & 0.2 & {$[26,21]$} \\
$\kappa_{v}$ & 1 & {$[26,21]$} & $\lambda$ & 0.5 & {$[26,21]$} \\
\hline
\end{tabular}

Table 1: Parameters used in the model.

tissue fibers -and degrade them), while the proliferating cells occupy the regions with very low tissue density. This is in agreement with the go-or-grow dichotomy and the degeneracy of the diffusion coefficient in equation ( $1 \mathrm{a}$ ): For $v=0$ (no tissue) the migrating cells stop and become proliferating cells. Moreover, the model is able to reproduce the often irregular shape of a tumor and the associated spread of cancer cells exhibiting various infiltrative (INF) patterns. According to the Japanese gastric association group [42], the latter provide a way to classify local invasiveness and tumor malignancy. In particular, Figure 2 exhibits some small 'islands' of cell aggregates, transiently isolated from the main tumor, which then grow and merge again with the neoplastic cell mass. That tumor cells have an infiltrative spread, form fingering patterns, and closely follow the specific tissue structure has been recognized for many types of cancer; perhaps the most prominent example featuring these characteristics are gliomas, see e.g. $[43,44,45,46]$. This behavior has also been confirmed by several models in a different mathematical framework, but still relying on the go-or-grow dichotomy and leading to related reaction-diffusion-taxis equations $[17,18]$. Like those models, the present setting allows to account for tumor heterogeneity w. r.t. the migratory/proliferative phenotypes of the constituent cells. As mentioned in the Introduction, this heterogeneity also reflects in the differentiated therapeutic response, an essential issue in therapy planning and assessment. Including therapy effects like e. g., in [21] can be easily addressed in this context as well. While current biomedical imaging only allows to determine the gross tumor volume, such models open the way to provide an (although imperfect) estimation of the tumor composition upon relying on the patient-specific tissue architecture and to correspondingly predict the extent of the neoplastic tissue.

We also compared the model (1) presented here to the previous model in [26], where no distinction has been made between the tumor cells (in moving and proliferative phenotypes, respectively). To this aim we simulated both models upon using the same parameters (from Table 1). The results are shown in Figure 3. The invasion speed of the tumor cells from the model (1) relying on the go-or-grow hypothesis is lower when compared to the model in [26]. This is due to the fact that in the model from [26] the whole cell population is simultaneously proliferating (generating tumor cell mass) and migrating, while in model (1) the cells defer migration for proliferating. This leads in model [26] to a higher cell mass at the invading tips, hence also to faster tumor advancement. The overall form of the predicted tumor remains, however, comparable, see Figure 3.

\section{Appendix A}

In this section we collect several auxiliary results on member-by-member products used above. We begin with a lemma which deals with the uniform integrability of member-by-member products.

Lemma A.1 (Uniform integrability for products) Let $\Omega$ be a measurable subset of $\mathbb{R}^{N}$ with finite measure and $I$ be some set. Let $\left\{f_{i}\right\}_{i \in I},\left\{g_{i}\right\}_{i \in I} \subset L^{2}(\Omega)$ be two families such that $\left\{\left|f_{i}\right|^{2}\right\}_{i \in I}$ is uniformly integrable and $\left\{g_{i}\right\}_{i \in I}$ is uniformly bounded in $L^{2}(\Omega)$. Then the family $\left\{f_{i} g_{i}\right\}_{i \in I}$ of member-by-member products is uniformly integrable.

This well-known property can be readily proved by using the definition of the uniform integrability. We leave the details to the reader. The following lemma is a generalization of the Lions lemma [47, Lemma 1.3] and the known result on weak-strong convergence for member-by-member products.

Lemma A.2 (Weak-a.e. convergence, [26]) Let $\Omega$ be a measurable subset of $\mathbb{R}^{N}$ with finite measure. Let $f, f_{n}: \Omega \rightarrow \mathbb{R}, n \in \mathbb{N}$ be measurable functions and $g, g_{n} \in L^{1}(\Omega), n \in \mathbb{N}$. Assume further that $f_{n} \underset{n \rightarrow \infty}{\rightarrow} f$ a.e. in $\Omega$ and $g_{n} \underset{n \rightarrow \infty}{\rightarrow} g, f_{n} g_{n} \underset{n \rightarrow \infty}{\rightarrow} \xi$ in $L^{1}(\Omega)$. Then, it holds that $\xi=f g$ a.e. in $\Omega$.

As was observed in [26], a similar result holds for sums of member-by-member products:

Lemma A.3 (Weak-a.e. convergence for sums, [26]) Let $\Omega$ be a measurable subset of $\mathbb{R}^{N}$ with finite measure and let $L \in \mathbb{N}$. Let $f^{l}, f_{n}^{l}: \Omega \rightarrow \mathbb{R}, n \in \mathbb{N}, l \in\{1, \ldots, L\}$, be measurable functions and $g^{l}, g_{n}^{l} \in L^{1}(\Omega), n \in \mathbb{N}, l \in\{1, \ldots, L\}$. Assume further that $f_{n}^{l} \underset{n \rightarrow \infty}{\rightarrow} f^{l}$ a.e. in $\Omega$ and $g_{n}^{l} \underset{n \rightarrow \infty}{\rightarrow} g^{l}, \sum_{l=1}^{L} f_{n}^{l} g_{n}^{l} \underset{n \rightarrow \infty}{\rightarrow} \xi$ in $L^{1}(\Omega)$. Then, it holds that $\xi=\sum_{l=1}^{L} f^{l} g^{l}$ a.e. in $\Omega$.

Remark A.4 Observe that, in Lemma A.3, it is not required that the sequences $\left\{f_{n}^{l} g_{n}^{l}\right\}_{n \in \mathbb{N}}$ themselves are convergent for $l \in\{1, \ldots, L\}$, but only their sum $\left\{\sum_{l=1}^{L} f_{n}^{l} g_{n}^{l}\right\}_{n \in \mathbb{N}}$. Thus, the result is applicable in the cases where the convergence of individual sequences is either false or unknown. 
Figure 1: Initial condition: densities of migrating $(M)$ and proliferating $(P)$ cells, tumor bulk $C=M+P$, and fiber network $V$. At start there are only proliferating cells located in a region with crossing fibers.
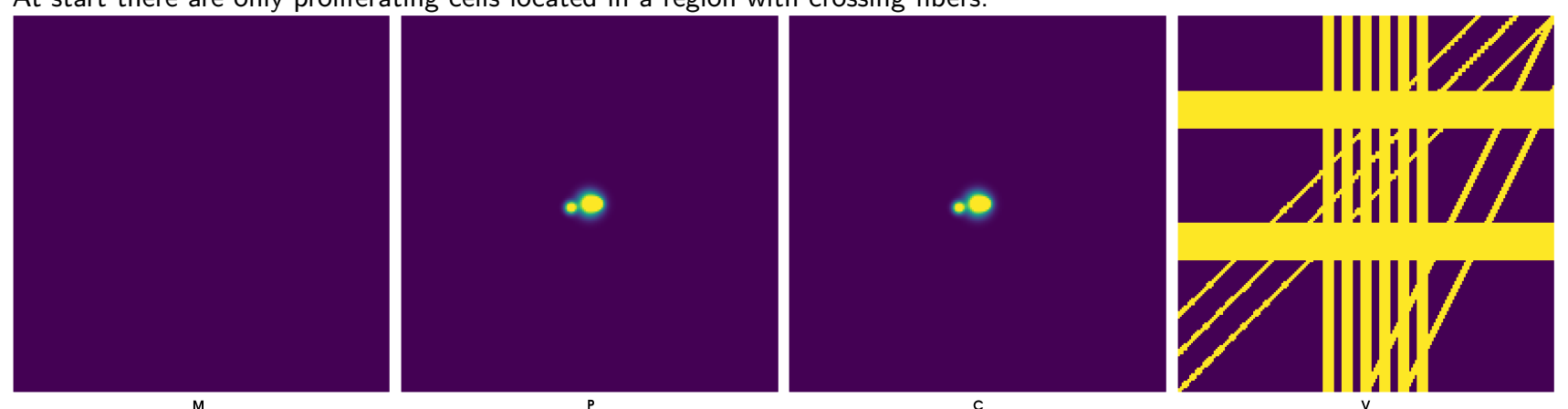

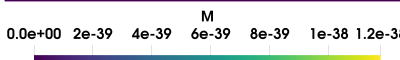

$0.0 \mathrm{e}+00$

$2^{P}$ $4.0 \mathrm{e}+00 \quad 0.0 \mathrm{e}+00$

$2^{\mathrm{C}}$

$\begin{array}{llll}5.00+00 & 5.00-02 \quad 0.2\end{array}$ 
Figure 2: Simulation results: densities of migrating $(M)$ and proliferating $(P)$ cells, tumor bulk $C=M+P$, and fiber network $V$. The proliferating tumor cells $(P)$ start to migrate when encountering fiber tracts, while the migrating cells $(M)$ follow the complex tissue (best seen in the left picture at time $t=10$ ). When the migrating cells come into contact with the lower horizontal stripe (at time $t=10$ ), they invade this stripe and start to degrade the fibers. When migrating cells encounter spaces between the fibers, they switch into the proliferating $(P)$ phenotype.
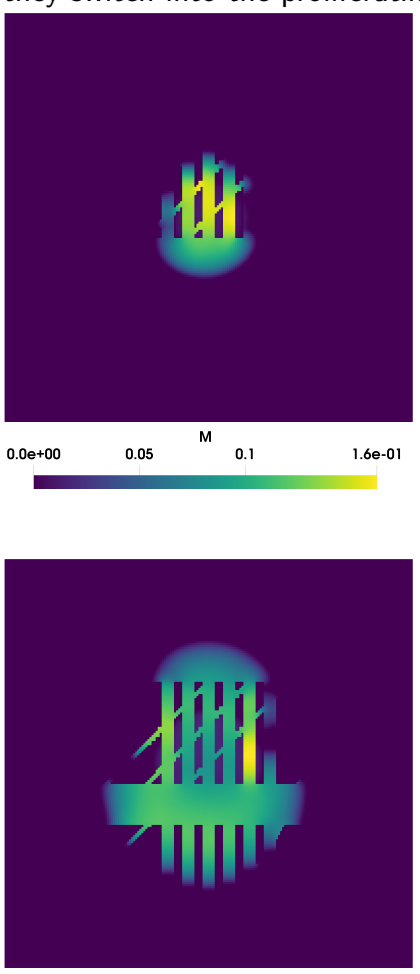

$\begin{array}{lllll}0.0 \mathrm{e}+00 & 0.05 & 0.1 & & \\ & & 0.15 & 0.22 .3 \mathrm{e}-0\end{array}$
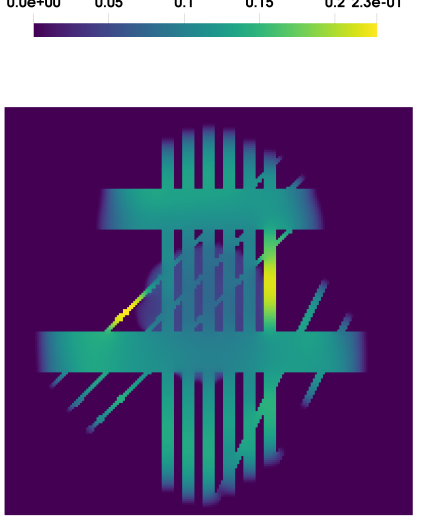

$\begin{array}{ccccccc}0.0 e+00 & 0.05 & 0.1 & 0.15 & 0.2 & 0.25 & 3.1 e-0\end{array}$
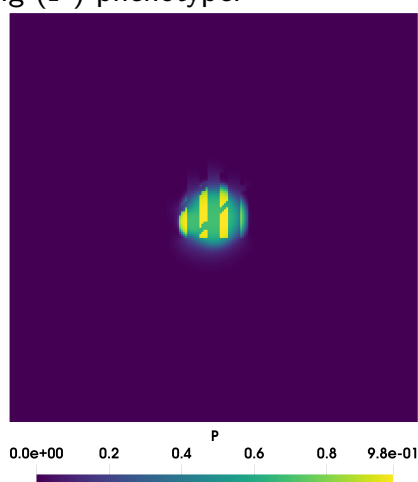

$\begin{array}{llll}0.8 & 9.8 \mathrm{e}-01 & 0.0 \mathrm{e}+00 & 0.2\end{array}$

(a) Simulation at time $t=10$
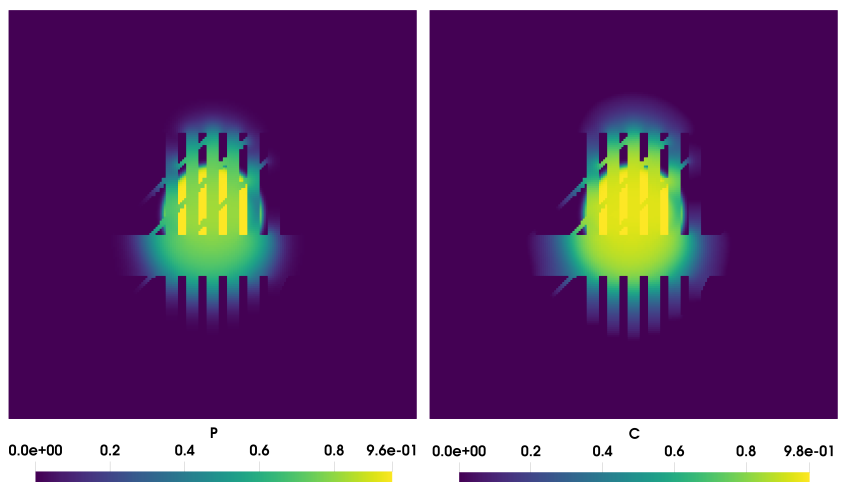

(b) Simulation at time $t=30$
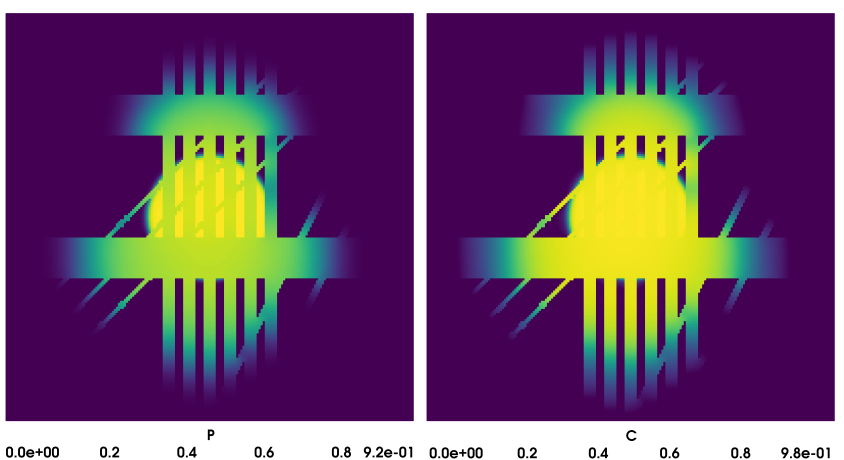

0.6

$\begin{array}{lll}0.8 & 9.2 \mathrm{e}-01 & 0.0 \mathrm{e}+00 \quad 0.2\end{array}$ 5

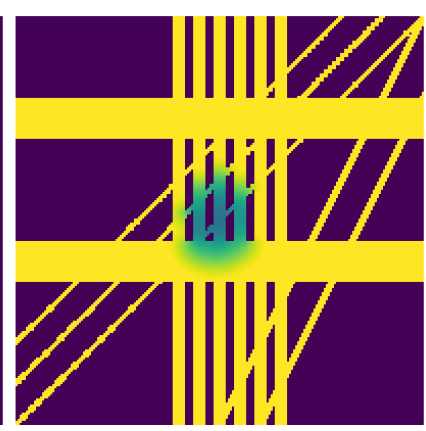

0.6 $0.8 \quad 1.0 \mathrm{e}+00$

e- $02 \quad 0.2$

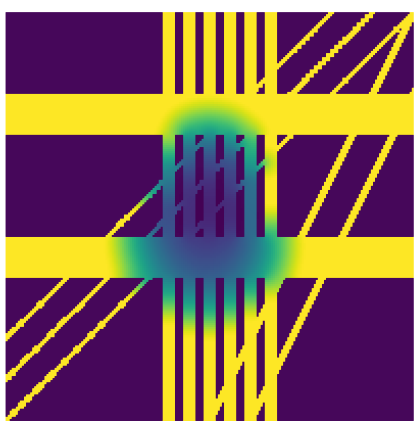

$0.8 \quad 1.0 e+00$

(c) Simulation at time $t=50$

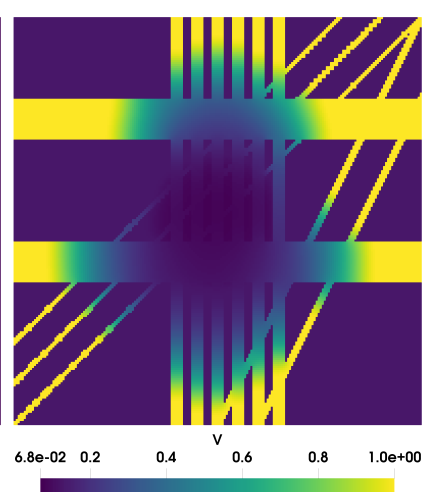


Figure 3: Comparison of model (1) to the model in [26]. The latter predicts a higher invasion speed (compare subfigures (a) and

(b)), however, the computed tumor shape is largely preserved, although at different time points (compare subfigures (c) and (d)).

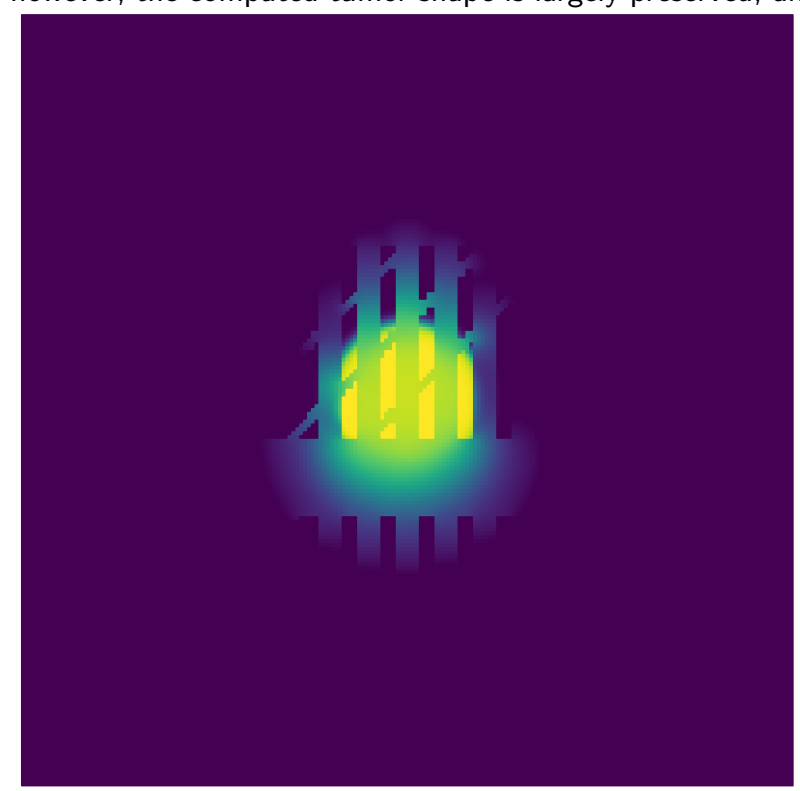

C

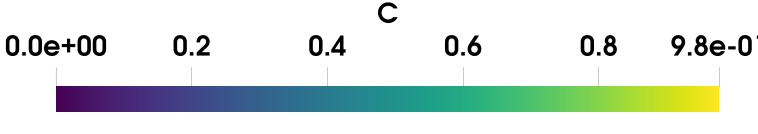

(a) Model (1) at time $t=20$

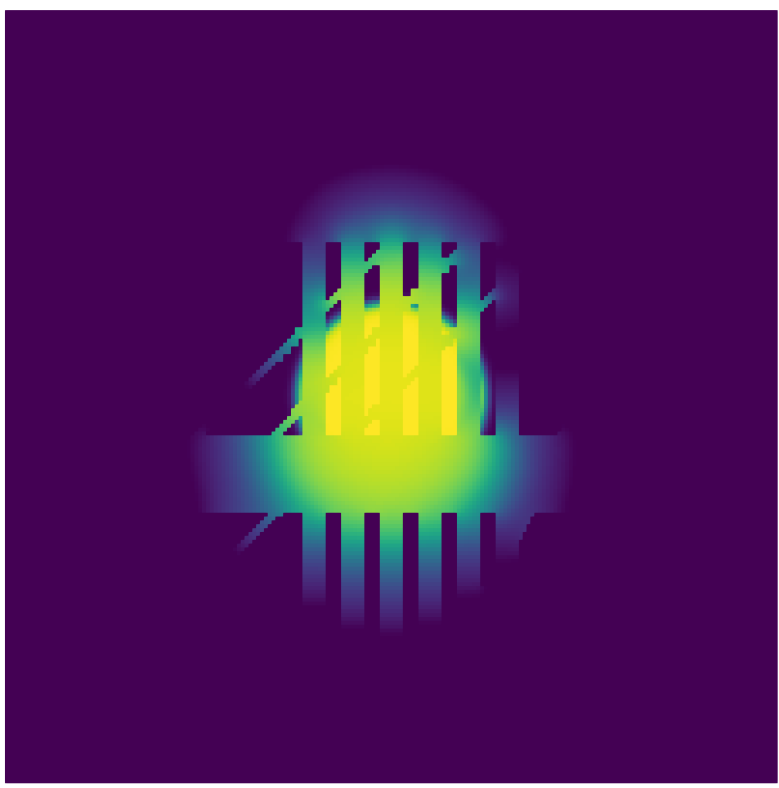

C

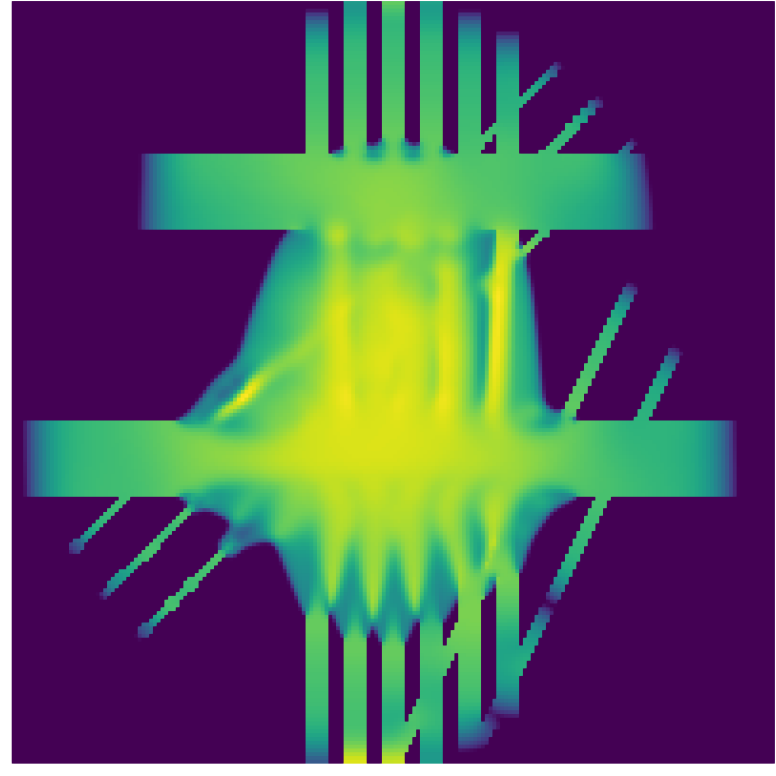

C

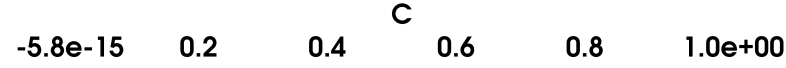

(b) Model in [26] at time $t=20$

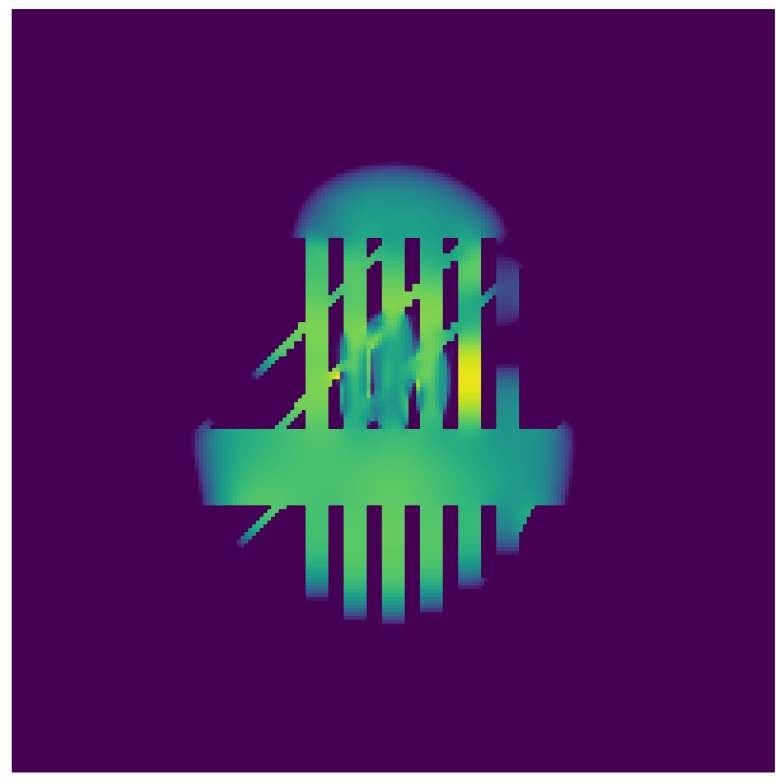

$0.0 e+00$ 0.2 0.4 0.6 $0.8 \quad 9.8 e-01 \quad-4.2 e-16$

0.2

0.4

0.6 $0.89 .0 e-01$

(c) Model (1) at time $t=30$

(d) Model in [26] at time $t=10$ 


\section{References}

1. Kleppe M, Levine R. Tumor heterogeneity confounds and illuminates: assessing the implications. Nature Medicine 2014; 20:342-344.

2. Marusyk A, Polyak K. Tumor heterogeneity: Causes and consequences. Biochimica et Biophysica Acta - Reviews on Cancer 2010; 1805(1):105-117.

3. Michor F, Weaver V. Understanding tissue context influences intratumor heterogeneity. Nature Cell Biol. 2014; 16:301-302.

4. Fidler I. Tumor heterogeneity and biology of cancer invasion and metastasis. Cancer Res. 1978; 38:2651-2660.

5. Heppner G. Tumor heterogeneity. Cancer Res. 1984; 44:2259-2265.

6. Giese A, Loo M, Tran N, Haskett D, Coons S, Berens M. Dichotomy of astrocytoma migration and proliferation. International Journal of Cancer 1996; 67:275-282.

7. Giese A, Bjerkvig R, Behrens M, Westphal M. Cost of migration: invasion of malignant gliomas and implications for treatment. J. Clin. Oncol. 2003; 21(8):1624-1636.

8. Jerby L, Wolf L, Denkert C, Stein G, Hilvo M, Oresic M, Geiger T, Ruppin E. Metabolic associations of reduced proliferation and oxidative stress in advanced breast cancer. Cancer Res. 2012; 72:5712-5720.

9. Widmer D, Hoek K, Cheng P, Eichhoff O, Biedermann T, et al. Hypoxia contributes to melanoma heterogeneity by triggering hif $1 \alpha-$ dependent phenotype switching. J. Invest. Dermat. 2013; 133:2436-2443.

10. Lefrank F, Brotchi J, Kiss R. Possible future issues in the treatment of glioblastomas: special emphasis on cell migration and the resistance of migrating glioblastoma cells to apoptosis. J. Clin. Oncol. 2005; 23:2411-2422.

11. Moore N, Houghton J, Lyle S. Slow-cycling therapy-resistant cancer cells. Stem Cells Dev. 2012; 21:1822-1830.

12. Steinbach J, Weller M. Apoptosis in gliomas: molecular mechanisms and therapeutic implications. J. NeuroOncol. 2004; 70:245-254.

13. Gerlee $\mathrm{P}$, Nelander $\mathrm{S}$. The impact of phenotypic switching on glioblastoma growth and invasion. PLOS Comp. Biol. $2012 ; 8$ :e1002 556.

14. Saut O, Lagaert J, Colin T, Fathallah-Shaykh H. A multilayer grow-or-go model for gbm: Effects of invasive cells and anti-angiogenesis on growth. Bull. Math. Biol. 2014; 76:2306-2333.

15. Fedotov S, lomin A. Migration and proliferation dichotomy in tumor-cell invasion. Phys. Rev. Lett. 2007; 98:118 101-1-118 101-4.

16. Chauvière A, Preziosi L, Byrne H. A model of cell migration within the extracellular matrix based on a phenotypic switching mechanism. Math. Med. Biol. 2010; 27:255-281.

17. Engwer C, Knappitsch M, Surulescu C. A multiscale model for glioma spread including cell-tissue interactions and proliferation. Math Biosc. Eng. 2016; 13:443-460.

18. Hunt A, Surulescu C. A multiscale modeling approach to glioma invasion with therapy. Vietnam Journal of Mathematics 2015; :1-20.

19. Meral G, Stinner C, Surulescu C. On a multiscale model involvig cell contractivity and its effects on tumor invasion. Disc. Cont. Dyn. Syst. B 2015; 20:189-213.

20. Meral G, Stinner C, Surulescu C. A multiscale model for acid-mediated tumor invasion: Therapy approaches. Journal of Coupled Systems and Multiscale Dynamics 2015; 3(2):135-142, doi:doi:10.1166/jcsmd.2015.1071.

21. Stinner C, Surulescu C, Uatay A. Global existence for a go-or-grow multiscale model for tumor invasion with therapy. Math. Models Methods Appl. Sci. 2016; 26:2163-2201.

22. Walker $\mathrm{C}$. Global existence for an age and spatially structured haptotaxis model with nonlinear age-boundary conditions. European Journal of Applied Mathematics 2008; 19(02):113-147.

23. Eberl HJ, Efendiev MA, Wrzosek D, Zhigun A. Analysis of a degenerate biofilm model with a nutrient taxis term. Discrete Contin. Dyn. Syst. 2014; 34(1):99-119, doi:10.3934/dcds.2014.34.99.

24. Laurençot P, Wrzosek D. A chemotaxis model with threshold density and degenerate diffusion, vol. 66. Chipot, M. and Escher, J. (ed.), Birkhäuser, Basel, 2005; 273-290.

25. Wang ZA, Winkler M, Wrzosek D. Global regularity versus infinite-time singularity formation in a chemotaxis model with volume-filling effect and degenerate diffusion. SIAM J. Math. Anal. 2012; 44(5):3502-3525, doi:10.1137/110853972.

26. Zhigun A, Surulescu C, Uatay A. Global existence for a degenerate haptotaxis model of cancer invasion. Zeitschrift für angewandte Mathematik und Physik 2016; 67(6):146, doi:10.1007/s00033-016-0741-0.

27. Winkler M, Surulescu C. Global weak solutions to a strongly degenerate haptotaxis model. In: Comm. Math. Sci. in print.

28. Li Y, Lankeit J. Boundedness in a chemotaxis? haptotaxis model with nonlinear diffusion. Nonlinearity 2016; 29(5):1564.

29. Tao Y, Winkler M. A chemotaxis-haptotaxis model: the roles of nonlinear diffusion and logistic source. SIAM J. Math. Anal. 2011; 43:685-705.

30. Zheng $\mathrm{P}, \mathrm{Mu} \mathrm{C}$, Song $\mathrm{X}$. On the boundedness and decay of solutions for a chemotaxis-haptotaxis system with nonlinear diffusion. Discr. Cont. Dyn. Syst. A 2016; 36:1737- 1757.

31. Bennett C, Sharpley R. Interpolation of Operators. Pure and Applied Mathematics, Elsevier Science, 1988.

32. Amann H. Nonhomogeneous linear and quasilinear elliptic and parabolic boundary value problems. Function spaces, differential operators and nonlinear analysis. Survey articles and communications of the international conference held in Friedrichsroda, Germany, September 20-26, 1992. Stuttgart: B. G. Teubner Verlagsgesellschaft, 1993; 9-126.

33. Evans LC, Gariepy RF. Measure theory and fine properties of functions. 2nd revised ed. 2nd revised edn., Boca Raton, FL: CRC Press, 2015.

34. Stinner C, Surulescu C, Winkler M. Global weak solutions in a PDE-ODE system modeling multiscale cancer cell invasion. SIAM J. Math. Anal. 2014; 46(3):1969-2007, doi:10.1137/13094058X.

35. Simon J. Compact sets in the space $L^{p}(0, T ; B)$. Ann. Mat. Pura Appl. (4) 1987; 146:65-96, doi:10.1007/BF01762360.

36. Blatt M, Burchardt A, Dedner A, Engwer C, Fahlke J, Flemisch B, Gersbacher C, GrÃ aser C, Gruber F, GrÃ $1 / 4$ ninger C, et al.. The distributed and unified numerics environment, version 2.4. Archive of Numerical Software 2016; 4(100):13-29.

37. Bastian P, Blatt M, Dedner A, Engwer C, Klöfkorn R, Ohlberger M, Sander O. A Generic Grid Interface for Parallel and Adaptive Scientific Computing. Part I: Abstract Framework. Computing 2008; 82(2-3):103-119, doi:10.1007/s00607-008-0003-x.

38. Bastian P, Blatt M, Dedner A, Engwer C, Klöfkorn R, Kornhuber R, Ohlberger M, Sander O. A Generic Grid Interface for Parallel and Adaptive Scientific Computing. Part II: Implementation and Tests in DUNE. Computing 2008; 82(2-3):121-138, doi: 10.1007/s00607-008-0004-9.

39. Blatt M, Bastian P. The iterative solver template library. Applied Parallel Computing. State of the Art in Scientific Computing, 
Lecture Notes in Computer Science, vol. 4699, Kågström B, Elmroth E, Dongarra J, Waśniewski J (eds.), Springer, 2007; 666-675, doi:10.1007/978-3-540-75755-9_82.

40. Blatt M, Bastian P. On the generic parallelisation of iterative solvers for the finite element method. Int. J. Comput. Sci. Engrg. 2008; 4(1):56-69, doi:10.1504/IJCSE.2008.021112.

41. Eymard R, Gallouet T, Herbin R. Finite Volume Methods. 2003

42. Association JGC. Japanese classification of gastric carcinoma: 3rd english edition. Gastric Cancer 2011; 14:10-112.

43. Coons S. Anatomy and growth patterns of diffuse gliomas. The gliomas, Berger M, Wilson C (eds.). W.B. Saunders Company, Philadelphia, 1999; 210-225.

44. Gerstner E, Chen PJ, Wen P, Jain R, Batchelor T, Sorensen G. Infiltrative patterns of glioblastoma spread detected via diffusion MRI after treatment with cediranib. Neuro-Oncology 2010; 12(5):466-472.

45. Giese A, Kluwe L, H M, E M, Westphal M. Migration of human glioma cells on myelin. Neurosurgery 1996; 38:755-764.

46. Giese A, Westphal M. Glioma invasion in the central nervous system. Neurosurgery 1996; 39:235-252.

47. Lions J. Quelques méthodes de résolution des problèmes aux limites non linéaires. Etudes mathematiques. Paris: Dunod; Paris: GauthierVillars. XX, 554 p. , 1969 